\title{
Supplementary material: Structural building principles of complex face-centered cubic intermetallics
}

\author{
Julia Dshemuchadse, Daniel Y. Jung and Walter Steurer*
}

Laboratory of Crystallography, Department of Materials, ETH Zurich, Wolfgang-Pauli-Strasse 10, 8093 Zurich, Switzerland

Correspondence e-mail: steurer@mat.ethz.ch

Submitted to Acta Crystallographica B, 14 February 2011

\section{Atomic sites - Superstructure description}

Table 1

Symmetry relations between basic and modulated structures.

\begin{tabular}{|c|c|c|c|c|c|}
\hline$F \overline{4} 3 m$ & $c F 16$ & $\rightarrow$ maximal non-isomorphic subgroup & $\vec{a}^{\prime}=3 \vec{a}$ & index $27 \rightarrow F \overline{4} 3 m$ & $c F 464$ \\
\hline$F d 3 m$ & $c F 16$ & $\begin{array}{l}\rightarrow \text { maximal non-isomorphic subgroup } \\
\quad\left(\& \text { origin shift }-\left(\frac{1}{4}, \frac{1}{4}, \frac{1}{4}\right) \text { with origin choice } 1\right)\end{array}$ & $\vec{a}^{\prime}=3 \vec{a}$ & index $27 \rightarrow F d 3 m$ & $c F 464$ \\
\hline$P \overline{4} 3 m$ & $c P 16$ & $\rightarrow$ maximal non-isomorphic subgroup (klassengleich) & $\vec{a}^{\prime}=2 \vec{a}$ & index $2 \rightarrow F \overline{4} 3 m$ & $c F 128$ \\
\hline$F \overline{4} 3 m$ & $c F 128$ & $\rightarrow$ maximal isomorphic subgroup (loss of centering) & - & $\rightarrow P \overline{4} 3 m$ & $c P 128$ \\
\hline$P \overline{4} 3 m$ & $c P 128$ & $\rightarrow$ maximal non-isomorphic subgroup (klassengleich) & $\vec{a}^{\prime \prime}=2 \vec{a}^{\prime}$ & $\rightarrow F \overline{4} 3 m$ & $c F 1024$ \\
\hline$P m \overline{3} m$ & $c P 16$ & $\rightarrow$ maximal non-isomorphic subgroup (klassengleich) & $\vec{a}^{\prime}=2 \vec{a}$ & index $2 \rightarrow F m \overline{3} m$ & $c F 128$ \\
\hline$F m \overline{3} m$ & $c F 128$ & $\rightarrow$ maximal isomorphic subgroup (loss of centering) & - & index $4 \rightarrow P n \overline{3} m$ & $c P 128$ \\
\hline $\operatorname{Pn} \overline{3} m$ & $c P 128$ & $\rightarrow$ maximal non-isomorphic subgroup (klassengleich) & $\vec{a}^{\prime \prime}=2 \vec{a}^{\prime}$ & index $2 \rightarrow F d \overline{3} m$ & $c F 1024$ \\
\hline$P m \overline{3} m$ & $c P 16$ & $\rightarrow$ maximal non-isomorphic subgroup (klassengleich) & $\vec{a}^{\prime}=2 \vec{a}$ & index $2 \rightarrow F m \overline{3} m$ & $c F 128$ \\
\hline$F m \overline{3} m$ & cF 128 & $\rightarrow$ maximal isomorphic subgroup (loss of centering) & - & $\rightarrow P m \overline{3} m$ & cP128 \\
\hline$P m \overline{3} m$ & $c P 128$ & $\rightarrow$ maximal non-isomorphic subgroup (klassengleich) & $\vec{a}^{\prime \prime}=2 \vec{a}^{\prime}$ & index $2 \rightarrow F m \overline{3} m$ & $c F 1024$ \\
\hline$P m \overline{3} m$ & $c P 16$ & $\rightarrow$ maximal non-isomorphic subgroup (klassengleich) & $\vec{a}^{\prime}=2 \vec{a}$ & index $2 \rightarrow F m \overline{3} m$ & $c F 128$ \\
\hline$F m \overline{3} m$ & $c F 128$ & $\rightarrow$ maximal isomorphic subgroup (loss of centering) & - & $\rightarrow P m \overline{3} m$ & $c P 128$ \\
\hline$P m \overline{3} m$ & $c P 128$ & $\rightarrow$ maximal non-isomorphic subgroup (klassengleich) & $\vec{a}^{\prime \prime}=2 \vec{a}^{\prime}$ & $\rightarrow F m \overline{3} c$ & $c F 1024$ \\
\hline$P m \overline{3}$ & $c P 16$ & $\rightarrow$ maximal non-isomorphic subgroup (klassengleich) & $\vec{a}^{\prime}=2 \vec{a}$ & $\rightarrow F m \overline{3}$ & $c F 128$ \\
\hline$F m \overline{3}$ & $c F 128$ & $\rightarrow$ maximal isomorphic subgroup (loss of centering) & & index 4 & $c P 128$ \\
\hline $\operatorname{Pn} \overline{3}$ & $c P 128$ & $\rightarrow$ maximal non-isomorphic subgroup (klassengleich) & $\vec{a}^{\prime \prime}=2 \vec{a}^{\prime}$ & index 2 & $c F 1024$ \\
\hline
\end{tabular}

The basic structures of all cubic compounds discussed in this work correspond to a $c F 16-\mathrm{NaTl}$ structure $(F d \overline{3} m, a b)$ or to derivatives of it, which exhibit lower symmetry.

The average structure of the $c F 464$-structures in $F \overline{4} 3 m$ preserve the space group symmetry the basic structure contains four Wyckoff positions, each one resulting from six Wyckoff positions of the original structure:

- site $1-4 a 0,0,0 \Leftarrow 4 a, 16 e 3,16 e 6,24 f 2,48 h 1,48 h 6$;

- site $2-4 c \frac{1}{4}, \frac{1}{4}, \frac{1}{4} \Leftarrow 4 d, 16 e 2,16 e 5,24 g 1,48 h 4,48 h 5$;

- site $3-4 b \frac{1}{2}, \frac{1}{2}, \frac{1}{2} \Leftarrow 4 b, 16 e 1,16 e 8,24 f 1,48 h 3,48 h 8$;

- site $4-4 d \frac{3}{4}, \frac{3}{4}, \frac{3}{4} \Leftarrow 4 c, 16 e 4,16 e 7,24 g 2,48 h 2,48 h 7$.

The average structure of the $c F 464$-structures in $F d \overline{3} m$ also exhibit the same symmetry as the original structures. The Wyckoff positions of the basic structure and the corresponding ones in the original unit cell are the following ${ }^{1}$ :

- site $1-8 a 0,0,0 \Leftarrow 8 b, 32 e 1,32 e 2,96 g 2,96 g 3$;

- site $2-8 b \frac{1}{2}, \frac{1}{2}, \frac{1}{2} \Leftarrow 8 a, 96 g 1,96 g 4$.

The average structure of $c F 1124-\mathrm{Cu}_{56.9} \mathrm{Cd}_{43.1}(F \overline{4} 3 m)$ has space group symmetry $P \overline{4} 3 m$. The atomic sites of the basic structure are: $1 a 0,0,0 ; 1 b \frac{1}{2}, \frac{1}{2}, \frac{1}{2} ; 3 c 0, \frac{1}{2}, \frac{1}{2} ; 3 d \frac{1}{2}, 0,0 ; 4 e x, x, x\left(x=\frac{1}{4}\right) ; 4 e x, x, x\left(x=\frac{3}{4}\right)$.

The average structures of $c F(1192-40)-\mathrm{Cd}_{66.7} \mathrm{Na}_{33.3}$ and $c F(1192-23)-\mathrm{Al}_{53.6} \mathrm{Mg}_{46.4}(F d \overline{3} m)$ has space group symmetry $P m \overline{3} m$. The atomic sites of the basic structure are: $1 a 0,0,0 ; 1 b \frac{1}{2}, \frac{1}{2}, \frac{1}{2} ; 3 c 0, \frac{1}{2}, \frac{1}{2} ; 3 d \frac{1}{2}, 0,0 ; 8 g x, x, x\left(x=\frac{1}{4}\right)$. The symmetry and sites are the same for the $(4 \times 4 \times 4)$-fold superstructures in space groups $F m \overline{3} m$ and $F m \overline{3} c$.

The average structure of the $(4 \times 4 \times 4)$-fold superstructure in space group $F d \overline{3}$ has space group symmetry $P m \overline{3}$. The atomic sites of the basic structure are: $1 a 0,0,0 ; 1 b \frac{1}{2}, \frac{1}{2}, \frac{1}{2} ; 3 c 0, \frac{1}{2}, \frac{1}{2} ; 3 d \frac{1}{2}, 0,0 ; 8 g x, x, x\left(x=\frac{1}{4}\right)^{2}$.

\footnotetext{
1 The classification of sites $96 \mathrm{~g} 2$ and $96 \mathrm{~g} 4$ is slightly ambiguous, since they have similar distances to both, sites 1 and 2 of the basic structure.

2 The coordinates of the Wyckoff positions are the same as in space group $P m \overline{3} m$ (see above), but their site symmetry is lower.
} 


\section{Symmetry relations}

\section{Table 2}

Details on the structure types between $c I 2-\mathrm{W}$ and the investigated complex intermetallic structures. Space groups in parentheses belong to the same lattice complex / exhibit the same Wyckoff coordinates.

\begin{tabular}{llcll}
\hline Structure type & Pearson symbol & Space group & Wyckoff sequence & Represented structures \\
\hline $\mathrm{W}$ & $c I 2$ & $\operatorname{Im} \overline{3} m$ & $a$ & $\operatorname{Im} \overline{3} m-c I 2,(P n \overline{3} m-c P 2)$ \\
$\mathrm{CsCl}$ & $c P 2$ & $P m \overline{3} m$ & $a b$ & $P m \overline{3} m-c P 2,(P \overline{4} 3 m-c P 2)$ \\
$\mathrm{Fe}_{13} \mathrm{Ge}_{3}{ }^{\dagger}$ & $c P 16$ & $P m \overline{3} m$ & $a b c d g$ & $P m \overline{3} m-c P 16,(P m \overline{3}-c P 16)$ \\
$\mathrm{CuHg}_{2} \mathrm{Ti}$ & $c F 16$ & $F \overline{4} m$ & $a b c d$ & $F \overline{4} 3 m-c F 16$ \\
$\mathrm{NaTl}$ & $c F 16$ & $F d \overline{3} m$ & $a b$ & $F d \overline{3} m-c F 16$ \\
$\mathrm{Ir}_{4} \mathrm{Sc}_{11}$ & $c F 120$ & $F m \overline{3} m$ & $a b d e f^{2}$ & $F m \overline{3} m-c F 128,(F m \overline{3}-c F 128)$ \\
$\mathrm{Ti}_{6} \mathrm{Co}_{7} \mathrm{Al}_{17}$ & $c F 120$ & $F \overline{4} 3 m$ & $a b e^{4} f g$ & $F \overline{4} 3 m-c F 128$ \\
\hline $\mathrm{Tl}_{7} \mathrm{Sb}_{2}$ & $c I 54$ & $I m \overline{3} m$ & $a e f h$ & $I m \overline{3} m-c I 54$ \\
$\mathrm{Al}_{4} \mathrm{Cu}_{9}$ & $c P 52$ & $P \overline{4} 3 m$ & $e^{4} f g i^{2}$ & $P \overline{4} 3 m-c P 54$ \\
$\mathrm{Cu}_{5} \mathrm{Zn}_{8}$ & $c I 52$ & $I \overline{4} 3 m$ & $\left(e^{2} g k\right)^{\ddagger}$ & $(P n \overline{3} m-c P 52)$ \\
\hline
\end{tabular}

$\dagger \mathrm{Fe}_{15} \mathrm{Co}$ and $\mathrm{Fe}_{9} \mathrm{Co}_{7}$ exhibit the same Wyckoff sequence with different occupations due to a differing composition.

$\ddagger$ These sites refer to space group $P n \overline{3} m$. The Wyckoff sequence in space group $I \overline{4} 3 m$ is $c^{2} e g$.

Table 3

Wyckoff sites in space group $\operatorname{Im} \overline{3} m$ of the Bärnighausen tree, $c I 2, c I 16$, cI432.

\begin{tabular}{rcrrrr}
\hline Site & Site symmetry & $x$ & $y$ & $z$ & Generating site \\
\hline \multicolumn{5}{c}{$\operatorname{Im} \overline{3} m,(229), c I 2, a \times a \times a-a$} \\
$2 a$ & $m \overline{3} m$ & 0 & 0 & \multicolumn{1}{c}{0} \\
\hline \multicolumn{5}{c}{$\operatorname{Im} \overline{3} m,(229), c I 16,2 a \times 2 a \times 2 a-a b c$} \\
$6 b$ & $m \overline{3} m$ & 0 & 0 & 0 & $1 a(\operatorname{Pm} \overline{3} m, c P 2)$ \\
$8 c$ & $4 / m m . m$ & 0 & $1 / 2$ & $1 / 2$ & $1 a(\operatorname{Pm} \overline{3} m, c P 2)$ \\
\hline \multicolumn{5}{c}{ Im $\overline{3} m,(229), c I 432,6 a \times 6 a \times 6 a-a b c e^{2} f^{2} g h^{2} i j k^{4}$} \\
$2 a$ & $m \overline{3} m$ & 0 & 0 & 0 & $2 a(\operatorname{Im} \overline{3} m, c I 16)$ \\
$6 b$ & $4 / m m . m$ & 0 & $1 / 2$ & $1 / 2$ & $6 b(\operatorname{Im} \overline{3} m, c I 16)$ \\
$8 c$ &.$\overline{3} m$ & $1 / 4$ & $1 / 4$ & $1 / 4$ & $8 c(\operatorname{Im} \overline{3} m, c I 16)$ \\
$12 e$ & $4 m . m$ & $1 / 3$ & 0 & 0 & $2 a(\operatorname{Im} \overline{3} m, c I 16)$ \\
$12 e$ & $4 m . m$ & $1 / 6$ & 0 & 0 & $6 b(\operatorname{Im} \overline{3} m, c I 16)$ \\
$16 f$ & $.3 m$ & $1 / 3$ & $1 / 3$ & $1 / 3$ & $2 a(\operatorname{Im} \overline{3} m, c I 16)$ \\
$16 f$ & $.3 m$ & $1 / 12$ & $1 / 12$ & $1 / 12$ & $8 c(\operatorname{Im} \overline{3} m, c I 16)$ \\
$24 g$ & $m m 2 .$. & $1 / 6$ & 0 & $1 / 2$ & $6 b(\operatorname{Im} \overline{3} m, c I 16)$ \\
$24 h$ & $m . m 2$ & $1 / 3$ & 0 & 0 & $2 a(\operatorname{Im} \overline{3} m, c I 16)$ \\
$24 h$ & $m . m 2$ & 0 & $1 / 6$ & $1 / 6$ & $6 b(\operatorname{Im} \overline{3} m, c I 16)$ \\
$48 i$ &. .2 & $1 / 4$ & $1 / 12$ & $5 / 12$ & $8 c(\operatorname{Im} \overline{3} m, c I 16)$ \\
$48 j$ & $m .$. & 0 & $1 / 6$ & $1 / 3$ & $6 b(\operatorname{Im} \overline{3} m, c I 16)$ \\
$48 k$ &..$m$ & $1 / 4$ & $1 / 4$ & $1 / 12$ & $8 c(\operatorname{Im} \overline{3} m, c I 16)$ \\
$48 k$ &..$m$ & $1 / 6$ & $1 / 6$ & $1 / 3$ & $6 b(\operatorname{Im} \overline{3} m, c I 16)$ \\
$48 k$ &..$m$ & $1 / 12$ & $1 / 12$ & $1 / 4$ & $8 c(\operatorname{Im} \overline{3} m, c I 16)$ \\
$48 k$ &..$m$ & $5 / 12$ & $5 / 12$ & $1 / 12$ & $8 c(\operatorname{Im} \overline{3} m, c I 16)$ \\
\hline
\end{tabular}

Table 4

Wyckoff sites in space group $P m \overline{3} m$ of the Bärnighausen tree, $c P 2, c P 16$, cP128.

\begin{tabular}{|c|c|c|c|c|c|}
\hline Site & Site symmetry & $x$ & $y$ & $z$ & Generating site \\
\hline & \multicolumn{5}{|c|}{$P m \overline{3} m,(221), c P 2, a \times a \times a-a b$} \\
\hline $1 a$ & $m \overline{3} m$ & 0 & 0 & 0 & $2 a(\operatorname{Im} \overline{3} m, c I 2)$ \\
\hline $1 b$ & $m \overline{3} m$ & $1 / 2$ & $1 / 2$ & $1 / 2$ & $2 a(\operatorname{Im} \overline{3} m, c I 2)$ \\
\hline \multicolumn{6}{|c|}{$P m \overline{3} m,(221), c P 16,2 a \times 2 a \times 2 a-a b c d g$} \\
\hline $1 a$ & $m \overline{3} m$ & 0 & 0 & 0 & $2 a(\operatorname{Im} \overline{3} m, c I 16)$ \\
\hline $1 b$ & $m \overline{3} m$ & $1 / 2$ & $1 / 2$ & $1 / 2$ & $2 a(\operatorname{Im} \overline{3} m, c I 16)$ \\
\hline $3 c$ & 4/mm.m & 0 & $1 / 2$ & $1 / 2$ & $6 b(\operatorname{Im} \overline{3} m, c I 16)$ \\
\hline $3 d$ & 4/mm.m & $1 / 2$ & 0 & 0 & $6 b(\operatorname{Im} \overline{3} m, c I 16)$ \\
\hline $8 g$ & $.3 m$ & $1 / 4$ & $1 / 4$ & $1 / 4$ & $8 c(\operatorname{Im} \overline{3} m, c I 16)$ \\
\hline \multicolumn{6}{|c|}{$P m \overline{3} m,(221), c P 128,4 a \times 4 a \times 4 a-a b c d e f g^{3} h_{j} m^{2}$} \\
\hline $1 a$ & $m \overline{3} m$ & 0 & 0 & 0 & $4 a(F m \overline{3} m, c F 128)$ \\
\hline $1 b$ & $m \overline{3} m$ & $1 / 2$ & $1 / 2$ & $1 / 2$ & $4 b(F m \overline{3} m, c F 128)$ \\
\hline $3 c$ & 4/mm.m & 0 & $1 / 2$ & $1 / 2$ & $4 a(F m \overline{3} m, c F 128)$ \\
\hline $3 d$ & 4/mm.m & $1 / 2$ & 0 & 0 & $4 b(F m \overline{3} m, c F 128)$ \\
\hline $6 e$ & $4 m . m$ & $1 / 4$ & 0 & 0 & $24 e(F m \overline{3} m, c F 128)$ \\
\hline $6 f$ & $4 m . m$ & $1 / 4$ & $1 / 2$ & $1 / 2$ & $24 e(F m \overline{3} m, c F 128)$ \\
\hline $8 g$ & $.3 m$ & $1 / 4$ & $1 / 4$ & $1 / 4$ & $8 c(F m \overline{3} m, c F 128)$ \\
\hline $8 g$ & $.3 m$ & $1 / 8$ & $1 / 8$ & $1 / 8$ & $32 f(F m \overline{3} m, c F 128)$ \\
\hline $8 g$ & $.3 m$ & $3 / 8$ & $3 / 8$ & $3 / 8$ & $32 f(F m \overline{3} m, c F 128)$ \\
\hline $12 h$ & $m m 2 .$. & $1 / 4$ & $1 / 2$ & 0 & $24 e(F m \overline{3} m, c F 128)$ \\
\hline $12 i$ & $m . m 2$ & 0 & $1 / 4$ & $1 / 4$ & $24 d(F m \overline{3} m, c F 128)$ \\
\hline $12 j$ & $m . m 2$ & $1 / 2$ & $1 / 4$ & $1 / 4$ & $24 d(F m \overline{3} m, c F 128)$ \\
\hline $24 m$ &..$m$ & $5 / 8$ & $5 / 8$ & $1 / 8$ & $32 f(F m \overline{3} m, c F 128)$ \\
\hline $24 m$ &..$m$ & $7 / 8$ & $7 / 8$ & $3 / 8$ & $32 f(F m \overline{3} m, c F 128)$ \\
\hline
\end{tabular}


Table 5

Wyckoff sites in space group $P \overline{4} 3 m$ of the Bärnighausen tree, $c P 2, c P 16$, $c P 128$.

\begin{tabular}{|c|c|c|c|c|c|}
\hline Site & Site symmetry & $x$ & $y$ & $z$ & Generating site \\
\hline & \multicolumn{5}{|c|}{$P 43 m,(215), c P 2, a \times a \times a-a b$} \\
\hline $1 a$ & $\overline{4} 3 m$ & 0 & 0 & 0 & $1 a(P m \overline{3} m, c P 2)$ \\
\hline $1 b$ & $\overline{4} 3 m$ & $1 / 2$ & $1 / 2$ & $1 / 2$ & $1 b(P m \overline{3} m, c P 2)$ \\
\hline \multicolumn{6}{|c|}{$P \overline{4} 3 m,(215), c P 16,2 a \times 2 a \times 2 a-a b c d e^{2}$} \\
\hline $1 a$ & $\overline{4} 3 m$ & 0 & 0 & 0 & $1 a(P m \overline{3} m, c P 16)$ \\
\hline $1 b$ & $\overline{4} 3 m$ & $1 / 2$ & $1 / 2$ & $1 / 2$ & $1 b(P m \overline{3} m, c P 16)$ \\
\hline $3 c$ & $\overline{4} 2 . m$ & 0 & $1 / 2$ & $1 / 2$ & $3 c(P m \overline{3} m, c P 16)$ \\
\hline $3 d$ & $\overline{4} 2 . m$ & $1 / 2$ & 0 & 0 & $3 d(P m \overline{3} m, c P 16)$ \\
\hline $4 e$ & $.3 m$ & $1 / 4$ & $1 / 4$ & $1 / 4$ & $8 g(P m \overline{3} m, c P 16)$ \\
\hline $4 e$ & $.3 m$ & $3 / 4$ & $3 / 4$ & $3 / 4$ & $8 g(P m \overline{3} m, c P 16)$ \\
\hline \multicolumn{6}{|c|}{$P \overline{4} 3 m,(215), c P 128,4 a \times 4 a \times 4 a-a b c d e^{6} f g h i^{6}$} \\
\hline $1 a$ & $\overline{4} 3 m$ & 0 & 0 & 0 & $1 a(P m \overline{3} m, c P 128)$ \\
\hline $1 b$ & $\overline{4} 3 m$ & $1 / 2$ & $1 / 2$ & $1 / 2$ & $1 b(P m \overline{3} m, c P 128)$ \\
\hline $3 c$ & $\overline{4} 2 . m$ & 0 & $1 / 2$ & $1 / 2$ & $3 c(P m \overline{3} m, c P 128)$ \\
\hline $3 d$ & $\overline{4} 2 . m$ & $1 / 2$ & 0 & 0 & $3 d(P m \overline{3} m, c P 128)$ \\
\hline $4 e$ & $.3 m$ & $1 / 4$ & $1 / 4$ & $1 / 4$ & $8 g(P m \overline{3} m, c P 128)$ \\
\hline $4 e$ & $.3 m$ & $3 / 4$ & $3 / 4$ & $3 / 4$ & $8 g(P m \overline{3} m, c P 128)$ \\
\hline $4 e$ & $.3 m$ & $1 / 8$ & $1 / 8$ & $1 / 8$ & $8 g(P m \overline{3} m, c P 128)$ \\
\hline $4 e$ & $.3 m$ & $3 / 8$ & $3 / 8$ & $3 / 8$ & $8 g(P m \overline{3} m, c P 128)$ \\
\hline $4 e$ & $.3 m$ & $5 / 8$ & $5 / 8$ & $5 / 8$ & $8 g(P m \overline{3} m, c P 128)$ \\
\hline $4 e$ & $.3 m$ & $7 / 8$ & $7 / 8$ & $7 / 8$ & $8 g(P m \overline{3} m, c P 128)$ \\
\hline $6 f$ & $2 . m m$ & $1 / 4$ & 0 & 0 & $6 e(P m \overline{3} m, c P 128)$ \\
\hline $6 g$ & $2 . \mathrm{mm}$ & $1 / 4$ & $1 / 2$ & $1 / 2$ & $6 f(P m \overline{3} m, c P 128)$ \\
\hline $12 h$ & $2 .$. & $1 / 4$ & $1 / 2$ & 0 & $12 h(P m \overline{3} m, c P 128)$ \\
\hline $12 i$ &..$m$ & $1 / 4$ & $1 / 4$ & 0 & $12 i(P m \overline{3} m, c P 128)$ \\
\hline $12 i$ &..$m$ & $1 / 4$ & $1 / 4$ & $1 / 2$ & $12 j(P m \overline{3} m, c P 128)$ \\
\hline $12 i$ &..$m$ & $5 / 8$ & $5 / 8$ & $1 / 8$ & $24 m(P m \overline{3} m, c P 128)$ \\
\hline $12 i$ &..$m$ & $7 / 8$ & $7 / 8$ & $3 / 8$ & $24 m(P m \overline{3} m, c P 128)$ \\
\hline $12 i$ &..$m$ & $1 / 8$ & $1 / 8$ & $5 / 8$ & $24 m(P m \overline{3} m, c P 128)$ \\
\hline $12 i$ &..$m$ & $3 / 8$ & $3 / 8$ & $7 / 8$ & $24 m(P m \overline{3} m, c P 128)$ \\
\hline
\end{tabular}

Table 6

Wyckoff sites in space group $P n \overline{3} m$ of the Bärnighausen tree, $c P 2, c P 16$, $c P 128$.

\begin{tabular}{rcrrrr}
\hline Site & Site symmetry & \multicolumn{1}{c}{$y$} & \multicolumn{1}{c}{$z$} & Generating site \\
\hline \multicolumn{5}{c}{$P n \overline{3} m,(224), c P 2, a \times a \times a-a$} \\
$2 a$ & $\overline{4} 3 m$ & 0 & 0 & 0 & $2 a(\operatorname{Im} \overline{3} m, c I 2)$ \\
\hline \multicolumn{7}{c}{$P n \overline{3} m,(224), c P 16,2 a \times 2 a \times 2 a-a b c d$} \\
$2 a$ & $\overline{4} 3 m$ & 0 & 0 & 0 & $2 a(\operatorname{Im} \overline{3} m, c I 16)$ \\
$4 b$ &.$\overline{3} m$ & $1 / 4$ & $1 / 4$ & $1 / 4$ & $8 c(\operatorname{Im} \overline{3} m, c I 16)$ \\
$4 c$ &.$\overline{3} m$ & $3 / 4$ & $3 / 4$ & $3 / 4$ & $8 c(\operatorname{Im} \overline{3} m, c I 16)$ \\
$6 d$ & $\overline{4} 2 . m$ & 0 & $1 / 2$ & $1 / 2$ & $6 b(\operatorname{Im} \overline{3} m, c I 16)$ \\
\hline \multicolumn{7}{c}{$P n \overline{3} m,(224), c P 128,4 a \times 4 a \times 4 a-a b c d e^{2} f g k^{3}$} \\
$2 a$ & $\overline{4} 3 m$ & 0 & 0 & 0 & $8 c(F m \overline{3} m, c F 128)$ \\
$4 b$ &.$\overline{3} m$ & $1 / 4$ & $1 / 4$ & $1 / 4$ & $4 a(F m \overline{3} m, c F 128)$ \\
$4 c$ &.$\overline{3} m$ & $3 / 4$ & $3 / 4$ & $3 / 4$ & $4 b(F m \overline{3} m, c F 128)$ \\
$6 d$ & $\overline{4} 2 . m$ & 0 & $1 / 2$ & $1 / 2$ & $8 c(F m \overline{3} m, c F 128)$ \\
$8 e$ & $.3 m$ & $3 / 8$ & $3 / 8$ & $3 / 8$ & $32 f(F m \overline{3} m, c F 128)$ \\
$8 e$ & $.3 m$ & $5 / 8$ & $5 / 8$ & $5 / 8$ & $32 f(F m \overline{3} m, c F 128)$ \\
$12 f$ & 2.22 & $1 / 4$ & 0 & $1 / 2$ & $24 d(F m \overline{3} m, c F 128)$ \\
$12 g$ & $2 . m m$ & $1 / 4$ & 0 & 0 & $24 d(F m \overline{3} m, c F 128)$ \\
$24 k$ &..$m$ & $1 / 4$ & $1 / 4$ & $1 / 2$ & $24 e(F m \overline{3} m, c F 128)$ \\
$24 k$ &..$m$ & $7 / 8$ & $7 / 8$ & $3 / 8$ & $32 f(F m \overline{3} m, c F 128)$ \\
$24 k$ &..$m$ & $1 / 8$ & $1 / 8$ & $5 / 8$ & $32 f(F m \overline{3} m, c F 128)$ \\
\hline \multicolumn{7}{c}{$2 k$} & & &
\end{tabular}

Table 7

Wyckoff sites in space group $P m \overline{3}$ of the Bärnighausen tree, $c P 16$.

\begin{tabular}{ccrrrr}
\hline Site & Site symmetry & $x$ & $y$ & $z$ & Generating site \\
\hline \multicolumn{5}{c}{$P m 3,(200), c P 16,2 a \times 2 a \times 2 a-a b c d i$} \\
$1 a$ & $m \overline{3}$. & 0 & 0 & 0 & $1 a(P m \overline{3} m, c P 16)$ \\
$1 b$ & $m \overline{3}$. & $1 / 2$ & $1 / 2$ & $1 / 2$ & $1 b(P m \overline{3} m, c P 16)$ \\
$3 c$ & $m m m .$. & 0 & $1 / 2$ & $1 / 2$ & $3 c(P m \overline{3} m, c P 16)$ \\
$3 d$ & $m m m .$. & $1 / 2$ & 0 & 0 & $3 d(P m \overline{3} m, c P 16)$ \\
$8 i$ & .3. & $1 / 4$ & $1 / 4$ & $1 / 4$ & $8 g(P m \overline{3} m, c P 16)$
\end{tabular}

Table 8

Wyckoff sites in space group $P n \overline{3}$ of the Bärnighausen tree, $c P 128$.

\begin{tabular}{rcrrrr}
\hline Site & Site symmetry & \multicolumn{1}{c}{$x$} & \multicolumn{2}{c}{$z$} & Generating site \\
\hline \multicolumn{5}{c}{$P n \overline{3},(201), c P 128,4 a \times 4 a \times 4 a-a b c d e^{2} f g h^{3}$} \\
$2 a$ & 23. & 0 & 0 & 0 & $8 c(F m \overline{3}, c F 128)$ \\
$4 b$ &.$\overline{3}$. & $1 / 4$ & $1 / 4$ & $1 / 4$ & $4 a(F m \overline{3}, c F 128)$ \\
$4 c$ &.$\overline{3}$. & $3 / 4$ & $3 / 4$ & $3 / 4$ & $4 b(F m \overline{3}, c F 128)$ \\
$6 d$ & $222 .$. & 0 & $1 / 2$ & $1 / 2$ & $8 c(F m \overline{3}, c F 128)$ \\
$8 e$ & .3. & $3 / 8$ & $3 / 8$ & $3 / 8$ & $32 f(F m \overline{3}, c F 128)$ \\
$8 e$ & .3. & $5 / 8$ & $5 / 8$ & $5 / 8$ & $32 f(F m \overline{3}, c F 128)$ \\
$12 f$ & $2 .$. & $1 / 4$ & 0 & 0 & $24 d(F m \overline{3}, c F 128)$ \\
$12 g$ & $2 .$. & $1 / 4$ & $1 / 2$ & 0 & $24 d(F m \overline{3}, c F 128)$ \\
$24 h$ & $2 .$. & $1 / 2$ & $1 / 4$ & $1 / 4$ & $24 e(F m \overline{3}, c F 128)$ \\
$24 h$ & $2 .$. & $3 / 8$ & $7 / 8$ & $7 / 8$ & $32 f(F m \overline{3}, c F 128)$ \\
$24 h$ & $2 .$. & $5 / 8$ & $1 / 8$ & $1 / 8$ & $32 f(F m \overline{3}, c F 128)$ \\
\hline
\end{tabular}

Table 9

Wyckoff sites in space group $F m \overline{3} m$ of the Bärnighausen tree, $c F 128$, $c F 1024$.

\begin{tabular}{|c|c|c|c|c|c|}
\hline Site & Site symm. & $x$ & $y$ & $z$ & Generating site \\
\hline & \multicolumn{5}{|c|}{$F m \overline{3} m,(225), c F 128,4 a \times 4 a \times 4 a-a b c d e f^{2}$} \\
\hline $4 a$ & $m \overline{3} m$ & 0 & 0 & 0 & $1 a(P m \overline{3} m, c P 16)$ \\
\hline $4 b$ & $m \overline{3} m$ & $1 / 2$ & $1 / 2$ & $1 / 2$ & $1 a(P m \overline{3} m, c P 16)$ \\
\hline $8 c$ & $\overline{4} 3 m$ & $1 / 4$ & $1 / 4$ & $1 / 4$ & $1 b(P m \overline{3} m, c P 16)$ \\
\hline $24 d$ & m.mm & 0 & $1 / 4$ & $1 / 4$ & $3 c(P m \overline{3} m, c P 16)$ \\
\hline $24 e$ & $4 m . m$ & $1 / 4$ & 0 & 0 & $3 d(P m \overline{3} m, c P 16)$ \\
\hline $32 f$ & $.3 m$ & $1 / 8$ & $1 / 8$ & $1 / 8$ & $8 g(P m \overline{3} m, c P 16)$ \\
\hline $32 f$ & $.3 m$ & $3 / 8$ & $3 / 8$ & $3 / 8$ & $8 g(P m \overline{3} m, c P 16)$ \\
\hline \multicolumn{6}{|c|}{$F m \overline{3} m,(225), c F 1024,8 a \times 8 a \times 8 a-a b c d e^{3} f^{6} h i^{2} j k^{5}$} \\
\hline $4 a$ & $m \overline{3} m$ & 0 & 0 & 0 & $1 a(P m \overline{3} m, c P 128)$ \\
\hline $4 b$ & $m \overline{3} m$ & $1 / 2$ & $1 / 2$ & $1 / 2$ & $1 a(P m \overline{3} m, c P 128)$ \\
\hline $8 c$ & $\overline{4} 3 m$ & $1 / 4$ & $1 / 4$ & $1 / 4$ & $1 b(P m \overline{3} m, c P 128)$ \\
\hline $24 d$ & m.mm & 0 & $1 / 4$ & $1 / 4$ & $3 c(P m \overline{3} m, c P 128)$ \\
\hline $24 e$ & $4 m . m$ & $1 / 4$ & 0 & 0 & $3 d(P m \overline{3} m, c P 128)$ \\
\hline $24 e$ & $4 m . m$ & $1 / 8$ & 0 & 0 & $6 e(P m \overline{3} m, c P 128)$ \\
\hline $24 e$ & $4 m . m$ & $3 / 8$ & 0 & 0 & $6 e(P m \overline{3} m, c P 128)$ \\
\hline $32 f$ & $.3 m$ & $1 / 8$ & $1 / 8$ & $1 / 8$ & $8 g(P m \overline{3} m, c P 128)$ \\
\hline $32 f$ & $.3 m$ & $3 / 8$ & $3 / 8$ & $3 / 8$ & $8 g(P m \overline{3} m, c P 128)$ \\
\hline $32 f$ & $.3 m$ & $1 / 16$ & $1 / 16$ & $1 / 16$ & $8 g(P m \overline{3} m, c P 128)$ \\
\hline $32 f$ & $.3 m$ & $3 / 16$ & $3 / 16$ & $3 / 16$ & $8 g(P m \overline{3} m, c P 128)$ \\
\hline $32 f$ & $.3 m$ & $9 / 16$ & $9 / 16$ & $9 / 16$ & $8 g(P m \overline{3} m, c P 128)$ \\
\hline $32 f$ & $.3 m$ & $11 / 16$ & $11 / 16$ & $11 / 16$ & $8 g(P m \overline{3} m, c P 128)$ \\
\hline $48 h$ & $m . m 2$ & 0 & $1 / 8$ & $1 / 8$ & $12 i(P m \overline{3} m, c P 128)$ \\
\hline $48 i$ & $m . m 2$ & $1 / 2$ & $1 / 8$ & $1 / 8$ & $12 i(P m \overline{3} m, c P 128)$ \\
\hline $48 i$ & $m . m 2$ & $1 / 8$ & $1 / 4$ & $1 / 4$ & $6 f(P m \overline{3} m, c P 128)$ \\
\hline $96 j$ & $m .$. & 0 & $1 / 8$ & $1 / 4$ & $12 h(P m \overline{3} m, c P 128)$ \\
\hline $96 k$ &..$m$ & $1 / 4$ & $1 / 8$ & $1 / 8$ & $12 j(P m \overline{3} m, c P 128)$ \\
\hline $96 k$ &..$m$ & $5 / 16$ & $5 / 16$ & $1 / 16$ & $24 m(P m \overline{3} m, c P 128)$ \\
\hline $96 k$ &..$m$ & $7 / 16$ & $7 / 16$ & $3 / 16$ & $24 m(P m \overline{3} m, c P 128)$ \\
\hline $96 k$ &..$m$ & $13 / 16$ & $13 / 16$ & $9 / 16$ & $24 m(P m \overline{3} m, c P 128)$ \\
\hline $96 k$ &..$m$ & $15 / 16$ & $15 / 16$ & $11 / 16$ & $24 m(P m \overline{3} m, c P 128)$ \\
\hline
\end{tabular}


Table 10

Wyckoff sites in space group $F m \overline{3} c$ of the Bärnighausen tree, $c F 1024$.

\begin{tabular}{rcrrrr}
\hline Site & Site symmetry & \multicolumn{1}{c}{$x$} & \multicolumn{1}{c}{$z$} & Generating site \\
\hline \multicolumn{5}{c}{$F m \overline{3} c,(226), c F$} & $1024,8 a \times 8 a \times 8 a-a b c d e f g^{3} h i^{2} j^{2}$ \\
$8 a$ & 432 & $1 / 4$ & $1 / 4$ & $1 / 4$ & $1 b(P m \overline{3} m, c P 128)$ \\
$8 b$ & $m \overline{3}$. & 0 & 0 & 0 & $1 a(P m \overline{3} m, c P 128)$ \\
$24 c$ & $\overline{4} m .2$ & $1 / 4$ & 0 & 0 & $3 d(P m \overline{3} m, c P 128)$ \\
$24 d$ & $4 / m .$. & 0 & $1 / 4$ & $1 / 4$ & $3 c(P m \overline{3} m, c P 128)$ \\
$48 e$ & $m m 2 .$. & $1 / 8$ & 0 & 0 & $6 e(P m \overline{3} m, c P 128)$ \\
$48 f$ & $4 .$. & $1 / 8$ & $1 / 2$ & $1 / 2$ & $6 f(P m \overline{3} m, c P 128)$ \\
$64 g$ & .3. & $1 / 8$ & $1 / 8$ & $1 / 8$ & $8 g(P m \overline{3} m, c P 128)$ \\
$64 g$ & .3. & $1 / 16$ & $1 / 16$ & $1 / 16$ & $8 g(P m \overline{3} m, c P 128)$ \\
$64 g$ & .3. & $3 / 16$ & $3 / 16$ & $3 / 16$ & $8 g(P m \overline{3} m, c P 128)$ \\
$96 h$ &. .2 & $1 / 4$ & $1 / 8$ & $1 / 8$ & $12 j(P m \overline{3} m, c P 128)$ \\
$96 i$ & $m .$. & 0 & $1 / 8$ & $1 / 4$ & $12 h(P m \overline{3} m, c P 128)$ \\
$96 i$ & $m .$. & 0 & $1 / 8$ & $1 / 8$ & $12 i(P m \overline{3} m, c P 128)$ \\
$192 j$ & 1 & $5 / 16$ & $5 / 16$ & $1 / 16$ & $24 m(P m \overline{3} m, c P 128)$ \\
$192 j$ & 1 & $7 / 16$ & $7 / 16$ & $3 / 16$ & $24 m(P m \overline{3} m, c P 128)$ \\
\hline
\end{tabular}

Table 11

Wyckoff sites in space group $F \overline{4} 3 m$ of the Bärnighausen tree, $c F 16$, $c F 128, c F 432$.

\begin{tabular}{|c|c|c|c|c|c|}
\hline Site & Site symmetry & $x$ & $y$ & $z$ & Generating site \\
\hline & \multicolumn{5}{|c|}{$F \overline{43 m},(216), c F 16,2 a \times 2 a \times 2 a-a b c d$} \\
\hline $4 a$ & $\overline{4} 3 m$ & 0 & 0 & 0 & $8 a(F d \overline{3} m, c F 16)$ \\
\hline $4 b$ & $\overline{4} 3 m$ & $1 / 2$ & $1 / 2$ & $1 / 2$ & $8 b(F d \overline{3} m, c F 16)$ \\
\hline $4 c$ & $\overline{4} 3 m$ & $1 / 4$ & $1 / 4$ & $1 / 4$ & $8 a(F d \overline{3} m, c F 16)$ \\
\hline $4 d$ & $\overline{4} 3 m$ & $3 / 4$ & $3 / 4$ & $3 / 4$ & $8 b(F d \overline{3} m, c F 16)$ \\
\hline \multicolumn{6}{|c|}{$F \overline{4} 3 m,(216), c F 128,4 a \times 4 a \times 4 a-a b c d e^{4} f g$} \\
\hline $4 a$ & $\overline{4} 3 m$ & 0 & 0 & 0 & $1 a(P \overline{4} 3 m, c P 16)$ \\
\hline $4 b$ & $\overline{4} 3 m$ & $1 / 2$ & $1 / 2$ & $1 / 2$ & $1 a(P \overline{4} 3 m, c P 16)$ \\
\hline $4 c$ & $\overline{4} 3 m$ & $1 / 4$ & $1 / 4$ & $1 / 4$ & $1 b(P \overline{4} 3 m, c P 16)$ \\
\hline $4 d$ & $\overline{4} 3 m$ & $3 / 4$ & $3 / 4$ & $3 / 4$ & $1 b(P \overline{4} 3 m, c P 16)$ \\
\hline $16 e$ & $.3 m$ & $1 / 8$ & $1 / 8$ & $1 / 8$ & $4 e(P \overline{4} 3 m, c P 16)$ \\
\hline $16 e$ & $.3 m$ & $3 / 8$ & $3 / 8$ & $3 / 8$ & $4 e(P \overline{4} 3 m, c P 16)$ \\
\hline $16 e$ & $.3 m$ & $5 / 8$ & $5 / 8$ & $5 / 8$ & $4 e(P \overline{4} 3 m, c P 16)$ \\
\hline $16 e$ & $.3 m$ & $7 / 8$ & $7 / 8$ & $7 / 8$ & $4 e(P \overline{4} 3 m, c P 16)$ \\
\hline $24 f$ & $2 . m m$ & $1 / 4$ & 0 & 0 & $3 d(P \overline{4} 3 m, c P 16)$ \\
\hline $24 g$ & $2 . \mathrm{mm}$ & 0 & $1 / 4$ & $1 / 4$ & $3 c(P \overline{4} 3 m, c P 16)$ \\
\hline \multicolumn{6}{|c|}{$F \overline{4} 3 m,(216), c F 432,6 a \times 6 a \times 6 a-a b c d e^{8} f^{2} g^{2} h^{4}$} \\
\hline $4 a$ & $\overline{4} 3 m$ & 0 & 0 & 0 & $4 a(F \overline{4} 3 m, c F 16)$ \\
\hline $4 b$ & $\overline{4} 3 m$ & $1 / 2$ & $1 / 2$ & $1 / 2$ & $4 b(F \overline{4} 3 m, c F 16)$ \\
\hline $4 c$ & $\overline{4} 3 m$ & $1 / 4$ & $1 / 4$ & $1 / 4$ & $4 c(F \overline{4} 3 m, c F 16)$ \\
\hline $4 d$ & $\overline{4} 3 m$ & $3 / 4$ & $3 / 4$ & $3 / 4$ & $4 d(F \overline{4} 3 m, c F 16)$ \\
\hline $16 e$ & $.3 m$ & $1 / 3$ & $1 / 3$ & $1 / 3$ & $4 a(F \overline{4} 3 m, c F 16)$ \\
\hline $16 e$ & $.3 m$ & $2 / 3$ & $2 / 3$ & $2 / 3$ & $4 a(F \overline{4} 3 m, c F 16)$ \\
\hline $16 e$ & $.3 m$ & $1 / 6$ & $1 / 6$ & $1 / 6$ & $4 b(F \overline{4} 3 m, c F 16)$ \\
\hline $16 e$ & $.3 m$ & $5 / 6$ & $5 / 6$ & $5 / 6$ & $4 b(F \overline{4} 3 m, c F 16)$ \\
\hline $16 e$ & $.3 m$ & $1 / 12$ & $1 / 12$ & $1 / 12$ & $4 d(F \overline{4} 3 m, c F 16)$ \\
\hline $16 e$ & $.3 m$ & $5 / 12$ & $5 / 12$ & $5 / 12$ & $4 d(F \overline{4} 3 m, c F 16)$ \\
\hline $16 e$ & $.3 m$ & $7 / 12$ & $7 / 12$ & $7 / 12$ & $4 c(F \overline{4} 3 m, c F 16)$ \\
\hline $16 e$ & $.3 m$ & $11 / 12$ & $11 / 12$ & $11 / 12$ & $4 c(F \overline{4} 3 m, c F 16)$ \\
\hline $24 f$ & $2 . \mathrm{mm}$ & $1 / 3$ & 0 & 0 & $4 a(F \overline{4} 3 m, c F 16)$ \\
\hline $24 f$ & 2. $\mathrm{mm}$ & $1 / 6$ & 0 & 0 & $4 b(F \overline{4} 3 m, c F 16)$ \\
\hline $24 g$ & $2 . \mathrm{mm}$ & $1 / 12$ & $1 / 4$ & $1 / 4$ & $4 d(F \overline{4} 3 m, c F 16)$ \\
\hline $24 g$ & $2 . \mathrm{mm}$ & $7 / 12$ & $1 / 4$ & $1 / 4$ & $4 c(F \overline{4} 3 m, c F 16)$ \\
\hline $48 h$ &..$m$ & $1 / 3$ & $1 / 3$ & 0 & $4 a(F \overline{4} 3 m, c F 16)$ \\
\hline $48 h$ &..$m$ & $1 / 6$ & $1 / 6$ & $1 / 2$ & $4 b(F \overline{4} 3 m, c F 16)$ \\
\hline $48 h$ &..$m$ & $5 / 12$ & $5 / 12$ & $1 / 4$ & $4 c(F \overline{4} 3 m, c F 16)$ \\
\hline $48 h$ &..$m$ & $1 / 12$ & $1 / 12$ & $3 / 4$ & $4 d(F \overline{4} 3 m, c F 16)$ \\
\hline
\end{tabular}

Table 12

Wyckoff sites in space group $F \overline{4} 3 m$ of the Bärnighausen tree, $c F 1024$.

\begin{tabular}{|c|c|c|c|c|c|}
\hline Site & Site symm. & $x$ & $y$ & $z$ & Generating site \\
\hline & \multicolumn{5}{|c|}{$F \overline{4} 3 m,(216), c F 1024,8 a \times 8 a \times 8 a-a b c d e^{12} f^{3} g^{3} h^{12} i$} \\
\hline $4 a$ & $\overline{4} 3 m$ & 0 & 0 & 0 & $1 a(P \overline{4} 3 m, c F 128)$ \\
\hline $4 b$ & $\overline{4} 3 m$ & $1 / 2$ & $1 / 2$ & $1 / 2$ & $1 a(P \overline{4} 3 m, c P 128)$ \\
\hline $4 c$ & $\overline{4} 3 m$ & $1 / 4$ & $1 / 4$ & $1 / 4$ & $1 b(P \overline{4} 3 m, c P 128)$ \\
\hline $4 d$ & $\overline{4} 3 m$ & $3 / 4$ & $3 / 4$ & $3 / 4$ & $1 b(P \overline{4} 3 m, c P 128)$ \\
\hline $16 e$ & $.3 m$ & $1 / 8$ & $1 / 8$ & $1 / 8$ & $4 e(P \overline{4} 3 m, c P 128)$ \\
\hline $16 e$ & $.3 m$ & $3 / 8$ & $3 / 8$ & $3 / 8$ & $4 e(P \overline{4} 3 m, c P 128)$ \\
\hline $16 e$ & $.3 m$ & $5 / 8$ & $5 / 8$ & $5 / 8$ & $4 e(P \overline{4} 3 m, c P 128)$ \\
\hline $16 e$ & $.3 m$ & $7 / 8$ & $7 / 8$ & $7 / 8$ & $4 e(P \overline{4} 3 m, c P 128)$ \\
\hline $16 e$ & $.3 m$ & $1 / 16$ & $1 / 16$ & $1 / 16$ & $4 e(P \overline{4} 3 m, c P 128)$ \\
\hline $16 e$ & $.3 m$ & $3 / 16$ & $3 / 16$ & $3 / 16$ & $4 e(P \overline{4} 3 m, c P 128)$ \\
\hline $16 e$ & $.3 m$ & $5 / 16$ & $5 / 16$ & $5 / 16$ & $4 e(P \overline{4} 3 m, c P 128)$ \\
\hline $16 e$ & $.3 m$ & $7 / 16$ & $7 / 16$ & $7 / 16$ & $4 e(P \overline{4} 3 m, c P 128)$ \\
\hline $16 e$ & $.3 m$ & $9 / 16$ & $9 / 16$ & $9 / 16$ & $4 e(P \overline{4} 3 m, c P 128)$ \\
\hline $16 e$ & $.3 m$ & $11 / 16$ & $11 / 16$ & $11 / 16$ & $4 e(P \overline{4} 3 m, c P 128)$ \\
\hline $16 e$ & $.3 m$ & $13 / 16$ & $13 / 16$ & $13 / 16$ & $4 e(P \overline{4} 3 m, c P 128)$ \\
\hline $16 e$ & $.3 m$ & $15 / 16$ & $15 / 16$ & $15 / 16$ & $4 e(P \overline{4} 3 m, c P 128)$ \\
\hline $24 f$ & $2 . \mathrm{mm}$ & $1 / 4$ & 0 & 0 & $3 d(P \overline{4} 3 m, c P 128)$ \\
\hline $24 f$ & $2 . \mathrm{mm}$ & $1 / 8$ & 0 & 0 & $6 f(P \overline{4} 3 m, c P 128)$ \\
\hline $24 f$ & $2 . \mathrm{mm}$ & $5 / 8$ & 0 & 0 & $6 f(P \overline{4} 3 m, c P 128)$ \\
\hline $24 g$ & $2 . \mathrm{mm}$ & 0 & $1 / 4$ & $1 / 4$ & $3 c(P \overline{4} 3 m, c P 128)$ \\
\hline $24 g$ & $2 . \mathrm{mm}$ & $1 / 8$ & $1 / 4$ & $1 / 4$ & $6 g(P \overline{4} 3 m, c P 128)$ \\
\hline $24 g$ & $2 . \mathrm{mm}$ & $5 / 8$ & $1 / 4$ & $1 / 4$ & $6 g(P \overline{4} 3 m, c P 128)$ \\
\hline $48 h$ &..$m$ & $1 / 8$ & $1 / 8$ & 0 & $12 i(P \overline{4} 3 m, c P 128)$ \\
\hline $48 h$ &..$m$ & $5 / 8$ & $5 / 8$ & $1 / 2$ & $12 i(P \overline{4} 3 m, c P 128)$ \\
\hline $48 h$ &..$m$ & $1 / 8$ & $1 / 8$ & $1 / 4$ & $12 i(P \overline{4} 3 m, c P 128)$ \\
\hline $48 h$ &..$m$ & $5 / 8$ & $5 / 8$ & $3 / 4$ & $12 i(P \overline{4} 3 m, c P 128)$ \\
\hline $48 h$ &..$m$ & $5 / 16$ & $5 / 16$ & $1 / 16$ & $12 i(P \overline{4} 3 m, c P 128)$ \\
\hline $48 h$ &..$m$ & $7 / 16$ & $7 / 16$ & $3 / 16$ & $12 i(P \overline{4} 3 m, c P 128)$ \\
\hline $48 h$ &..$m$ & $1 / 16$ & $1 / 16$ & $5 / 16$ & $12 i(P \overline{4} 3 m, c P 128)$ \\
\hline $48 h$ &..$m$ & $3 / 16$ & $3 / 16$ & $7 / 16$ & $12 i(P \overline{4} 3 m, c P 128)$ \\
\hline $48 h$ &..$m$ & $13 / 16$ & $13 / 16$ & $9 / 16$ & $12 i(P \overline{4} 3 m, c P 128)$ \\
\hline $48 h$ &..$m$ & $15 / 16$ & $15 / 16$ & $11 / 16$ & $12 i(P \overline{4} 3 m, c P 128)$ \\
\hline $48 h$ &..$m$ & $9 / 16$ & $9 / 16$ & $13 / 16$ & $12 i(P \overline{4} 3 m, c P 128)$ \\
\hline $48 h$ &..$m$ & $11 / 16$ & $11 / 16$ & $15 / 16$ & $12 i(P \overline{4} 3 m, c P 128)$ \\
\hline $96 i$ & 1 & $1 / 8$ & $1 / 4$ & 0 & $12 h(P \overline{4} 3 m, c P 128)$ \\
\hline
\end{tabular}

Table 13

Wyckoff sites in space group $F d \overline{3} m$ of the Bärnighausen tree, $c F 16$, $c F 432$.

\begin{tabular}{rcrrrr}
\hline Site & Site symmetry & $x$ & $y$ & \multicolumn{1}{c}{$z$} & Generating site \\
\hline \multicolumn{5}{c}{$F d \overline{3} m,(227), c F 16,2 a \times 2 a \times 2 a-a b$} \\
$8 a$ & $\overline{4} 3 m$ & 0 & 0 & 0 & $2 a(P n \overline{3} m, c P 2)$ \\
$8 b$ & $\overline{4} 3 m$ & $1 / 2$ & $1 / 2$ & $1 / 2$ & $2 a(P n \overline{3} m, c P 2)$ \\
\hline \multicolumn{5}{c}{$F d \overline{3} m,(227), c F 432,6 a \times 6 a \times 6 a-a b e^{4} f^{2} g^{2}$} \\
$8 a$ & $\overline{4} 3 m$ & 0 & 0 & 0 & $8 a(F d \overline{3} m, c F 16)$ \\
$8 b$ & $\overline{4} 3 m$ & $1 / 2$ & $1 / 2$ & $1 / 2$ & $8 b(F d \overline{3} m, c F 16)$ \\
$32 e$ & $.3 m$ & $1 / 3$ & $1 / 3$ & $1 / 3$ & $8 a(F d \overline{3} m, c F 16)$ \\
$32 e$ & $.3 m$ & $1 / 12$ & $1 / 12$ & $1 / 12$ & $8 b(F d \overline{3} m, c F 16)$ \\
$32 e$ & $.3 m$ & $5 / 12$ & $5 / 12$ & $5 / 12$ & $8 b(F d \overline{3} m, c F 16)$ \\
$32 e$ & $.3 m$ & $7 / 12$ & $7 / 12$ & $7 / 12$ & $8 a(F d \overline{3} m, c F 16)$ \\
$48 f$ & $2 . m m$ & $1 / 3$ & 0 & 0 & $8 a(F d \overline{3} m, c F 16)$ \\
$48 f$ & $2 . m m$ & $5 / 6$ & 0 & 0 & $8 b(F d \overline{3} m, c F 16)$ \\
$96 g$ &. .2 & $7 / 12$ & $7 / 12$ & $1 / 4$ & $8 a(F d \overline{3} m, c F 16)$ \\
$96 g$ &. .2 & $1 / 12$ & $1 / 12$ & $3 / 4$ & $8 b(F d \overline{3} m, c F 16)$ \\
\hline
\end{tabular}


Table 14

Wyckoff sites in space group $F d \overline{3} m$ of the Bärnighausen tree, $c F 1024$.

\begin{tabular}{rcrrrr}
\hline Site & Site symm. & \multicolumn{1}{c}{$x$} & \multicolumn{2}{c}{$z$} & Generating site \\
\hline & $F d \overline{3} m,(227), c F 1024,8 a \times 8 a \times 8 a-a b c d e^{5} f^{3} g^{6} h$ \\
$8 a$ & $\overline{4} 3 m$ & 0 & 0 & 0 & $2 a(P n \overline{3} m, c P 128)$ \\
$8 b$ & $\overline{4} 3 m$ & $1 / 2$ & $1 / 2$ & $1 / 2$ & $2 a(P n \overline{3} m, c P 128)$ \\
$16 c$ &.$\overline{3} m$ & $1 / 8$ & $1 / 8$ & $1 / 8$ & $4 b(P n \overline{3} m, c P 128)$ \\
$16 d$ &.$\overline{3} m$ & $5 / 8$ & $5 / 8$ & $5 / 8$ & $4 b(P n \overline{3} m, c P 128)$ \\
$32 e$ & $.3 m$ & $3 / 8$ & $3 / 8$ & $3 / 8$ & $4 c(P n \overline{3} m, c P 128)$ \\
$32 e$ & $.3 m$ & $3 / 16$ & $3 / 16$ & $3 / 16$ & $8 e(P n \overline{3} m, c P 128)$ \\
$32 e$ & $.3 m$ & $5 / 16$ & $5 / 16$ & $5 / 16$ & $8 e(P n \overline{3} m, c P 128)$ \\
$32 e$ & $.3 m$ & $11 / 16$ & $11 / 16$ & $11 / 16$ & $8 e(P n \overline{3} m, c P 128)$ \\
$32 e$ & $.3 m$ & $13 / 16$ & $13 / 16$ & $13 / 16$ & $8 e(P n \overline{3} m, c P 128)$ \\
$48 f$ & $2 . m m$ & $1 / 4$ & 0 & 0 & $6 d(P n \overline{3} m, c P 128)$ \\
$48 f$ & $2 . m m$ & $1 / 8$ & 0 & 0 & $12 g(P n \overline{3} m, c P 128)$ \\
$48 f$ & $2 . m m$ & $5 / 8$ & 0 & 0 & $12 g(P n \overline{3} m, c P 128)$ \\
$96 g$ &..$m$ & $1 / 8$ & $1 / 8$ & $1 / 4$ & $24 k(P n \overline{3} m, c P 128)$ \\
$96 g$ &..$m$ & $5 / 8$ & $5 / 8$ & $3 / 4$ & $24 k(P n \overline{3} m, c P 128)$ \\
$96 g$ &..$m$ & $7 / 16$ & $7 / 16$ & $3 / 16$ & $24 k(P n \overline{3} m, c P 128)$ \\
$96 g$ &..$m$ & $1 / 16$ & $1 / 16$ & $5 / 16$ & $24 k(P n \overline{3} m, c P 128)$ \\
$96 g$ &..$m$ & $15 / 16$ & $15 / 16$ & $11 / 16$ & $24 k(P n \overline{3} m, c P 128)$ \\
$96 g$ &..$m$ & $9 / 16$ & $9 / 16$ & $13 / 16$ & $24 k(P n \overline{3} m, c P 128)$ \\
$96 h$ &. .2 & $1 / 8$ & 0 & $1 / 4$ & $12 f(P n \overline{3} m, c P 128)$ \\
\hline
\end{tabular}

\section{Table 15}

Wyckoff sites in space group $F m \overline{3}$ of the Bärnighausen tree, $c F 128$.

\begin{tabular}{rcrrrr}
\hline Site & Site symmetry & \multicolumn{1}{c}{$x$} & \multicolumn{1}{c}{$y$} & $z$ & Generating site \\
\hline \multicolumn{5}{c}{$F m \overline{3},(202), c F 128,4 a \times 4 a \times 4 a-a b c d e f^{2}$} \\
$4 a$ & $m \overline{3}$. & 0 & 0 & 0 & $1 a(P m \overline{3}, c P 16)$ \\
$4 b$ & $m \overline{3}$. & $1 / 2$ & $1 / 2$ & $1 / 2$ & $1 a(P m \overline{3}, c P 16)$ \\
$8 c$ & 23. & $1 / 4$ & $1 / 4$ & $1 / 4$ & $1 b(P m \overline{3}, c P 16)$ \\
$24 d$ & $2 / m .$. & 0 & $1 / 4$ & $1 / 4$ & $3 c(P m \overline{3}, c P 16)$ \\
$24 e$ & $m m 2 .$. & $1 / 4$ & 0 & 0 & $3 d(P m \overline{3}, c P 16)$ \\
$32 f$ & .3. & $1 / 8$ & $1 / 8$ & $1 / 8$ & $8 i(P m \overline{3}, c P 16)$ \\
$32 f$ & .3. & $3 / 8$ & $3 / 8$ & $3 / 8$ & $8 i(P m \overline{3}, c P 16)$ \\
\hline
\end{tabular}

Table 16

Wyckoff sites in space group $F d \overline{3}$ of the Bärnighausen tree, $c F 1024$.

\begin{tabular}{rcrrrr}
\hline Site & Site symm. & \multicolumn{1}{c}{$x$} & \multicolumn{1}{c}{$z$} & Generating site \\
\hline & $F d \overline{3},(203), c F 1024,4 a \times 4 a \times 4 a-a b c d e^{5} f^{3} g^{7}$ \\
$8 a$ & 23. & 0 & 0 & 0 & $2 a(P n \overline{3}, c P 128)$ \\
$8 b$ & 23. & $1 / 2$ & $1 / 2$ & $1 / 2$ & $2 a(P n \overline{3}, c P 128)$ \\
$16 c$ &.$\overline{3}$. & $1 / 8$ & $1 / 8$ & $1 / 8$ & $4 b(P n \overline{3}, c P 128)$ \\
$16 d$ &.$\overline{3}$. & $5 / 8$ & $5 / 8$ & $5 / 8$ & $4 b(P n \overline{3}, c P 128)$ \\
$32 e$ & .3. & $3 / 8$ & $3 / 8$ & $3 / 8$ & $4 c(P n \overline{3}, c P 128)$ \\
$32 e$ & .3. & $3 / 16$ & $3 / 16$ & $3 / 16$ & $8 e(P n \overline{3}, c P 128)$ \\
$32 e$ & .3. & $5 / 16$ & $5 / 16$ & $5 / 16$ & $8 e(P n \overline{3}, c P 128)$ \\
$32 e$ & .3. & $11 / 16$ & $11 / 16$ & $11 / 16$ & $8 e(P n \overline{3}, c P 128)$ \\
$32 e$ & .3. & $13 / 16$ & $13 / 16$ & $13 / 16$ & $8 e(P n \overline{3}, c P 128)$ \\
$48 f$ & $2 .$. & $1 / 4$ & 0 & 0 & $6 d(P n \overline{3}, c P 128)$ \\
$48 f$ & $2 .$. & $1 / 8$ & 0 & 0 & $12 f(P n \overline{3}, c P 128)$ \\
$48 f$ & $2 .$. & $5 / 8$ & 0 & 0 & $12 f(P n \overline{3}, c P 128)$ \\
$96 g$ & 1 & $1 / 8$ & $1 / 4$ & 0 & $12 g(P n \overline{3}, c P 128)$ \\
$96 g$ & 1 & $1 / 4$ & $1 / 8$ & $1 / 8$ & $24 h(P n \overline{3}, c P 128)$ \\
$96 g$ & 1 & $3 / 4$ & $5 / 8$ & $5 / 8$ & $24 h(P n \overline{3}, c P 128)$ \\
$96 g$ & 1 & $3 / 16$ & $7 / 16$ & $7 / 16$ & $32 f(P n \overline{3}, c P 128)$ \\
$96 g$ & 1 & $5 / 16$ & $1 / 16$ & $1 / 16$ & $32 f(P n \overline{3}, c P 128)$ \\
$96 g$ & 1 & $11 / 16$ & $15 / 16$ & $15 / 16$ & $32 f(P n \overline{3}, c P 128)$ \\
$96 g$ & 1 & $13 / 16$ & $9 / 16$ & $9 / 16$ & $32 f(P n \overline{3}, c P 128)$ \\
\hline
\end{tabular}

Table 17

Wyckoff sites in the additional space groups of the inset to the Bärnighausen tree, $\operatorname{Im} \overline{3} m-c I 54, P m \overline{3} m-c P 54, P \overline{4} 3 m-c P 54, P n \overline{3} m$ $-c P 54$.

\begin{tabular}{|c|c|c|c|c|c|}
\hline Site & Site symmetry & $x$ & $y$ & $z$ & Generating site \\
\hline & \multicolumn{5}{|c|}{$\operatorname{Im} \overline{3} m,(229), c I 54,3 a \times 3 a \times 3 a-a e f h$} \\
\hline $2 a$ & $m \overline{3} m$ & 0 & 0 & 0 & $2 a(\operatorname{Im} \overline{3} m, c I 2)$ \\
\hline $12 e$ & $4 m . m$ & $1 / 3$ & 0 & 0 & $2 a(\operatorname{Im} \overline{3} m, c I 2)$ \\
\hline $16 f$ & $.3 m$ & $1 / 3$ & $1 / 3$ & $1 / 3$ & $2 a(\operatorname{Im} \overline{3} m, c I 2)$ \\
\hline $24 h$ & $m . m 2$ & 0 & $1 / 3$ & $1 / 3$ & $2 a(\operatorname{Im} \overline{3} m, c I 2)$ \\
\hline \multicolumn{6}{|c|}{$P m \overline{3} m,(221), c P 54,3 a \times 3 a \times 3 a-a b e f g^{2} i j$} \\
\hline $1 a$ & $m \overline{3} m$ & 0 & 0 & 0 & $1 a(P m \overline{3} m, c P 2)$ \\
\hline $1 b$ & $m \overline{3} m$ & $1 / 2$ & $1 / 2$ & $1 / 2$ & $1 b(P m \overline{3} m, c P 2)$ \\
\hline $6 e$ & $4 m . m$ & $1 / 3$ & 0 & 0 & $1 a(P m \overline{3} m, c P 2)$ \\
\hline $6 f$ & $4 m . m$ & $1 / 6$ & $1 / 2$ & $1 / 2$ & $1 b(P m \overline{3} m, c P 2)$ \\
\hline $8 g$ & $.3 m$ & $1 / 3$ & $1 / 3$ & $1 / 3$ & $1 a(P m \overline{3} m, c P 2)$ \\
\hline $8 g$ & $.3 m$ & $1 / 6$ & $1 / 6$ & $1 / 6$ & $1 b(P m \overline{3} m, c P 2)$ \\
\hline $12 i$ & $m . m 2$ & 0 & $1 / 3$ & $1 / 3$ & $1 a(P m \overline{3} m, c P 2)$ \\
\hline $12 j$ & $m . m 2$ & $1 / 2$ & $1 / 6$ & $1 / 6$ & $1 b(P m \overline{3} m, c P 2)$ \\
\hline \multicolumn{6}{|c|}{$P \overline{4} 3 m,(215), c P 54,3 a \times 3 a \times 3 a-a b e^{4} f g i^{2}$} \\
\hline $1 a$ & $\overline{4} 3 m$ & 0 & 0 & 0 & $1 a(P m \overline{3} m, c P 54)$ \\
\hline $1 b$ & $\overline{4} 3 m$ & $1 / 2$ & $1 / 2$ & $1 / 2$ & $1 b(P m \overline{3} m, c P 54)$ \\
\hline $4 e$ & $.3 m$ & $1 / 3$ & $1 / 3$ & $1 / 3$ & $8 g(P m \overline{3} m, c P 54)$ \\
\hline $4 e$ & $.3 m$ & $2 / 3$ & $2 / 3$ & $2 / 3$ & $8 g(P m \overline{3} m, c P 54)$ \\
\hline $4 e$ & $.3 m$ & $1 / 6$ & $1 / 6$ & $1 / 6$ & $8 g(P m \overline{3} m, c P 54)$ \\
\hline $4 e$ & $.3 m$ & $5 / 6$ & $5 / 6$ & $5 / 6$ & $8 g(P m \overline{3} m, c P 54)$ \\
\hline $6 f$ & 2.mm & $1 / 3$ & 0 & 0 & $6 e(P m \overline{3} m, c P 54)$ \\
\hline $6 g$ & $2 . \mathrm{mm}$ & $1 / 6$ & $1 / 2$ & $1 / 2$ & $6 f(P m \overline{3} m, c P 54)$ \\
\hline $12 i$ &..$m$ & $1 / 3$ & $1 / 3$ & 0 & $12 i(P m \overline{3} m, c P 54)$ \\
\hline $12 i$ &..$m$ & $1 / 6$ & $1 / 6$ & $1 / 2$ & $12 j(P m \overline{3} m, c P 54)$ \\
\hline \multicolumn{6}{|c|}{$P n \overline{3} m,(224), c P 54,3 a \times 3 a \times 3 a-a e^{2} g k$} \\
\hline $2 a$ & $\overline{4} 3 m$ & 0 & 0 & 0 & $2 a(P n \overline{3} m, c P 2)$ \\
\hline $8 e$ & $.3 m$ & $1 / 3$ & $1 / 3$ & $1 / 3$ & $2 a(P n \overline{3} m, c P 2)$ \\
\hline $8 e$ & $.3 m$ & $2 / 3$ & $2 / 3$ & $2 / 3$ & $2 a(P n \overline{3} m, c P 2)$ \\
\hline $12 g$ & $2 . m m$ & $1 / 3$ & 0 & 0 & $2 a(P n \overline{3} m, c P 2)$ \\
\hline $24 k$ &..$m$ & $1 / 3$ & $1 / 3$ & 0 & $2 a(P n \overline{3} m, c P 2)$ \\
\hline
\end{tabular}

The Wyckoff sites of the two largest structures in space group $F \overline{4} 3 m$, also shown in the Bärnighausen tree as $c F 5488$ and $c F 21296$, are not given. They may be derived using the International Tables for Crystallography - Volume A1: Symmetry Relations Between Space Group (Ed.s Hans Wondratschek and Ulrich Müller, 2006). 


\section{Atomic sites - Coordinates}

The sites belonging to the different clusters in cubic complex intermetallic structures in space group $F \overline{4} 3 m$ are given in Table 18 . The ones of the hypothetic subtype V are given in Table 19.

\section{Table 18}

Main cluster found in the structure of cubic complex intermetallics of space group $F \overline{4} 3 m$. Given are the sites belonging to a certain cluster (shell), the number of atoms, and the polyhedron built up by them. The following shells correspond to special clusters: 1 st shell, 1 st variant $-(14$ atoms $)$ rhombic dodecahedron, 1st shell, 2nd variant $-F K_{16}^{28} /$ Friauf polyhedron, 2nd shell $-F K_{40}^{76} /$ Frank-Kasper polyhedron with 76 faces and 40 vertices, 3rd shell, 6th variant $-F_{76}^{40} /$ fullerene polyhedron with 40 faces and 76 vertices. The subtypes I..IV in which the respective clusters occur are indicated in columns $S T$.

$\begin{aligned} & \text { Nr. of } \\ & \text { atoms }\end{aligned}$
$\begin{aligned} & \text { Cluster center } \\ & 1\end{aligned}$ 4a

Table 19

Main cluster found in the hypothetical structure of subtype $\mathrm{V}$ of cubic complex intermetallics in space group $F \overline{4} 3 \mathrm{~m}$. The clusters which do not occur in the above-discussed other subtypes are highlighted by bold letters. Given are the sites belonging to a certain cluster (shell), the number of atoms, and the polyhedron built up by them.

\begin{tabular}{|c|c|c|c|c|c|c|}
\hline $\begin{array}{l}\text { Cluster } \\
\text { shell }\end{array}$ & Polyhedron & $\begin{array}{l}\text { Nr. of } \\
\text { atoms }\end{array}$ & $(0,0,0)$ & $\begin{array}{l}\text { Sites around } \\
\left(\frac{1}{4}, \frac{1}{4}, \frac{1}{4}\right)\end{array}$ & $\begin{array}{l}\text { luster center } \\
\left(\frac{1}{2}, \frac{1}{2}, \frac{1}{2}\right)\end{array}$ & $\left(\frac{3}{4}, \frac{3}{4}, \frac{3}{4}\right)$ \\
\hline Center & - & 1 & $4 a$ & $4 c$ & $4 b$ & $4 d$ \\
\hline $1 \mathrm{st}$ & $F K_{16}^{28}$ & 16 & $16 \mathrm{e} 4,48 \mathrm{~h} 8$ & $16 e 1,48 h 5$ & $16 e 2,48 h 6$ & $16 e 3,48 h 7$ \\
\hline 2nd & $F K_{40}^{76}$ & $\begin{array}{l}40 \\
72\end{array}$ & $16 e 1,48 h 1,2,4$ & $\begin{array}{l}16 e 2,48 h 1,2,3 \\
\mathbf{4 8 h 4} 6 \mathbf{8}\end{array}$ & $\begin{array}{l}16 e 3,48 h 2,3,4 \\
\mathbf{4 8 h 1} \mathbf{5 . 7 . 8}\end{array}$ & $\begin{array}{l}16 e 4,48 h 1,3,4 \\
\mathbf{4 8 h 2} \mathbf{5 . 6 . 8}\end{array}$ \\
\hline
\end{tabular}

\section{Table 20}

Development of the third cluster shells throughout the subtypes I $\cdots \mathrm{V}(S T)$ with number of vertices and different faces $(t$ triangular, $q$ quadrangular, $p$ pentagonal, $h$ hexagon) for the sites of highest symmetry $4 a \cdots d\left(000, \frac{1}{2} \frac{1}{2} \frac{1}{2}, \frac{1}{4} \frac{1}{4} \frac{1}{4}\right.$, and $\frac{3}{4} \frac{3}{4} \frac{3}{4}$, respectively).

\begin{tabular}{ll|llll|llll}
\hline & \multicolumn{6}{|c|}{ No. of vertices } & \multicolumn{5}{c}{ No. of different faces } \\
ST & $4 a$ & $4 c$ & $4 b$ & $4 d$ & $4 a$ & $4 c$ & $4 b$ & $4 d$ \\
\hline I & 58 & 58 & 58 & 58 & $t^{4} q^{12} p^{12} h^{12}$ & $t^{4} q^{12} p^{12} h^{12}$ & $t^{4} q^{12} p^{12} h^{12}$ & $t^{4} q^{12} p^{12} h^{12}$ \\
II & 64 & 70 & 58 & 54 & $t^{4} p^{24} h^{12}$ & $p^{24} h^{16}$ & $t^{4} q^{12} p^{12} h^{12}$ & $t^{8} q^{12} p^{24}$ \\
III & 60 & 76 & 70 & 54 & $t^{8} p^{36}$ & $p^{12} h^{28}$ & $p^{24} h^{16}$ & $t^{8} q^{12} p^{24}$ \\
IV & 72 & 76 & 66 & 60 & $t^{4} p^{24} h^{16}$ & $p^{12} h^{28}$ & $t^{4} p^{36} h^{4}$ & $t^{8} p^{36}$ \\
\hline V & 72 & 72 & 72 & 72 & $t^{4} p^{24} h^{16}$ & $t^{4} p^{24} h^{16}$ & $t^{4} p^{24} h^{16}$ & $t^{4} p^{24} h^{16}$ \\
\hline
\end{tabular}

The sites of the clusters in structures in space group $F d \overline{3} m$ are listed in Table 21 . The sites of the one-shell clusters used in the modular explanation of the $c F 464$-structures in space group $F d \overline{3} m$ are given in Table 22 .

\section{Table 21}

Clusters found in the structure of cubic complex intermetallics of space group $F d \overline{3} m$. Given are the sites belonging to a certain cluster (shell), the number of atoms, and the polyhedron built up by them.

\begin{tabular}{|c|c|c|c|c|c|c|c|}
\hline \multirow{2}{*}{$\begin{array}{l}\text { Cluster } \\
\text { shell }\end{array}$} & \multicolumn{3}{|c|}{ Polyhedron } & \multicolumn{3}{|c|}{ Capped polyhedron } & \multirow[t]{2}{*}{ Atoms } \\
\hline & Shape & Atoms & Sites & Shape & Add. atoms & Additional sites & \\
\hline Center & - & 1 & $8 a$ & & & & 1 \\
\hline $1 \mathrm{st}$ & $t t$ & 12 & $12 \times 96 g 2$ & $F K_{16}^{28}$ & +4 & $+4 \times 32 e 1$ & 16 \\
\hline 2nd & $F_{28}^{16}$ & 28 & $4 \times 32 e 2,24 \times 96 g 1$ & $F K_{44}^{84}$ & +16 & $+4 \times 32 e 1,12 \times 96 g 3$ & 40 \\
\hline 3rd & $F_{84}^{44}$ & 84 & $24 \times 96 g 2,24 \times 96 g 3,36 \times 96 g 4$ & & & & \\
\hline $\begin{array}{l}\text { Center } \\
1 \text { st } \\
2 \text { nd }\end{array}$ & dodecahedron & 0 & $\begin{array}{l}16 d \\
2 \times 8 b, 6 \times 32 e 2,12 \times 96 g 1\end{array}$ & icosahedron & 12 & $6 \times 96 g 3,6 \times 96 g 4$ & 0 \\
\hline
\end{tabular}


Table 22

Atomic sites of one-shell clusters used in the modular explanation of cubic complex intermetallics of space group $F d \overline{3} m$. Given are the sites belonging to the cluster around a specified cluster center, the number of atoms, and the polyhedron built up by them.

\begin{tabular}{llrl}
\hline Center & Polyhedron & Atoms & Sites \\
\hline $8 a$ & truncated tetrahedron & 12 & $12 \times 96 g 2$ \\
& Friauf polyhedron & $16+4 \times 32 e 1$ \\
\hline \multirow{2}{*}{$32 e 2$} & truncated tetrahedron & 12 & $3 \times 96 g 2,3 \times 96 g 3,6 \times 96 g 4$ \\
& Friauf polyhedron & $16+3 \times 96 g 1,1 \times 8 b$ \\
\hline \multirow{2}{*}{$96 g 1$} & truncated tetrahedron & 12 & $2 \times 32 e 1,4 \times 96 g 2,4 \times 96 g 3,2 \times 96 g 4$ \\
& Friauf polyhedron & $16+3 \times 96 g 1,1 \times 32 e 1$ \\
\hline \multirow{2}{*}{$16 d$} & icosahedron & 12 & $6 \times 96 g 3,6 \times 96 g 4$ \\
\hline & truncated tetrahedron & 12 & $12 \times 96 g 4$ \\
& Friauf polyhedron & $16+4 \times 32 e 2$ \\
\hline
\end{tabular}

The sites of both structure types can be directly related to one another as shown in Table 23 . The site symmetry is preserved except for $32 e / 16 e$ sites, which have symmetry.$\overline{3} m$ in space group $F d \overline{3} m$ and $.3 m$ in $F \overline{4} 3 m$. Not listed are the $24 f$ and $24 g$ sites, which contribute to rhombic dodecahedra around $\overline{4} 3 m$ sites, which do not occur in the $F d \overline{3} m$ structures. Correspondingly, only half of the $16 e$ sites are related to the $32 e$ sites of space group $F d \overline{3} \mathrm{~m}$. Of sites $96 \mathrm{~g} 3$ and $96 \mathrm{~g} 4$, only half of the positions could be related to sites in $F \overline{4} 3 m$ structures: $48 h 5$ is part of site $96 g 2$, as $48 h 6$ relates directly to $96 g 4$. When we investigate the relationship between the structures it becomes clear that the inner cluster shells, which have the shape of Friauf polyhedra, should contain sites $16 e 3$ and $16 e 4$ instead of $16 e 5$ and $16 e 8$. They can be regarded as being oriented conversely and accordingly, not sites $48 h 7$ and $48 h 8$ are occupied but their counterparts, corresponding to half of the positions described by sites $96 \mathrm{~g} 3$ and $96 \mathrm{~g} 4$ in space group $F d \overline{3} m$. Therein, one half of the polyhedra have an opposite orientation with respect to the other half, whereas in the $F \overline{4} 3 m$ structures, all sub-units are oriented similarly.

Table 23

Relations of the atomic sites in cubic complex intermetallics of space groups $F d \overline{3} m$ and $F \overline{4} 3 m$.

\begin{tabular}{lllll}
\hline & & $F d \overline{3} m$ & $F \overline{4} 3 m$ \\
& Site & Site symmetry & Site & Site symmetry \\
\hline $8 a$ & $\overline{4} 3 m$ & $4 a, 4 c$ & $\overline{4} 3 m$ \\
$8 b$ & $\overline{4} 3 m$ & $4 b, 4 d$ & $\overline{4} 3 m$ \\
\hline $32 e 1$ & $.3 m$ & $16 e 1,16 e 5$ & $.3 m$ \\
& $32 e 2$ &.$\overline{3} m$ & $16 e 2,16 e 8$ & $.3 m$ \\
\hline $96 g 1$ &..$m$ & $48 h 1,48 h 2$ &..$m$ \\
& $96 g 2$ &..$m$ & $48 h 3,48 h 4$ &..$m$ \\
\hline $96 g 3$ &..$m$ & $48 h 5 \& .$. &..$m$ \\
& $96 g 4$ &..$m$ & $48 h 6 \& .$. &..$m$ \\
\hline
\end{tabular}




\section{Atomic sites - Occupations}

Table 24

Site occupations of all discussed complex intermetallic structures in space group $F \overline{4} 3 m$. A + denotes that a site is occupied, a - that it is empty. Sites labelled with different letters are occupied the following modifications: $\mathrm{m}$ - mixed occupation, $\mathrm{d}$ - deficient occupation, $\mathrm{s}-\mathrm{split}$ site. The $\mathrm{In}_{54.5} \mathrm{Pd}_{29.3} \mathrm{Ce}_{16.3}$ structure is subject to two specific features: the $16 e 1$ site (marked with a 2 ) is replaced by two sites $16 e$ whose average corresponds to the $16 e 1$ position. The $48 h 3$ site is not occupied but instead a (split) site $96 i$.

\begin{tabular}{|c|c|c|c|c|c|c|c|c|c|c|c|c|c|c|c|c|c|c|c|c|c|c|c|}
\hline \multirow[b]{2}{*}{ Structure } & \multicolumn{4}{|c|}{4} & \multicolumn{8}{|c|}{$16 \mathrm{e}$} & \multicolumn{2}{|c|}{$24 f$} & \multicolumn{2}{|c|}{$24 g$} & \multicolumn{7}{|c|}{$48 h$} \\
\hline & $a$ & $b$ & $c$ & $d$ & 1 & 2 & 3 & 4 & 5 & 6 & 7 & 8 & 1 & 2 & 1 & 2 & 1 & 2 & 3 & 4 & 5 & 6 & 7 \\
\hline \multicolumn{24}{|c|}{ Subtype I (10 structures) } \\
\hline theoretical & + & + & + & + & + & + & + & + & + & + & + & + & + & + & + & + & + & + & + & + & - & - & - \\
\hline $\mathrm{Li}_{64.3} \operatorname{In}_{26.5} \mathrm{Ag}_{9.2}$ & + & $\mathrm{m}$ & + & $\mathrm{m}$ & + & + & + & + & $\mathrm{m}$ & + & + & $\mathrm{m}$ & + & + & $\mathrm{m}$ & + & + & + & $\mathrm{m}$ & $\mathrm{m}$ & - & - & - \\
\hline $\mathrm{Li}_{81.0} \mathrm{~Pb}_{19.0}$ & + & - & - & - & + & + & + & + & + & + & + & + & + & + & + & + & + & + & + & + & - & - & - \\
\hline $\mathrm{Li}_{81.0} \mathrm{Sn}_{19.0}$ & + & - & - & - & + & + & + & + & + & + & + & + & + & + & + & + & + & + & + & + & - & - & - \\
\hline $\mathrm{Li}_{81.0} \mathrm{Ge}_{19.0}$ & $\mathrm{~d}$ & - & - & - & + & + & + & + & $\mathrm{s}$ & + & + & + & + & + & + & + & + & + & + & + & - & - & - \\
\hline $\mathrm{Zn}_{80.9} \mathrm{Pd}_{14.5} \mathrm{Al}_{4.6}$ & - & - & - & - & + & + & $\mathrm{s}$ & + & $\mathrm{m}$ & + & + & $\mathrm{m}$ & + & + & + & $\mathrm{m}$ & + & + & + & $\mathrm{m}$ & - & - & - \\
\hline $\mathrm{Zn}_{82.7} \mathrm{Pt}_{17.3}$ & - & - & - & - & $\mathrm{ms}$ & + & + & $\mathrm{m}$ & + & ds & + & + & $\mathrm{m}$ & $\mathrm{d}$ & $\mathrm{m}$ & $\mathrm{d}$ & + & d & + & + & - & - & - \\
\hline $\mathrm{Zn}_{81.6} \mathrm{Pt}_{18.4}$ & - & - & - & - & $\mathrm{s}$ & + & + & $\mathrm{m}$ & + & ds & + & + & $\mathrm{m}$ & + & $\mathrm{m}$ & + & + & $\mathrm{d}$ & + & + & - & - & - \\
\hline $\mathrm{Zn}_{78.7} \mathrm{Pd}_{16.0} \mathrm{Al}_{5.3}$ & - & - & - & - & + & + & ds & + & $\mathrm{m}$ & + & + & $\mathrm{d}$ & + & + & + & $\mathrm{m}$ & + & + & + & $\mathrm{m}$ & - & - & - \\
\hline $\mathrm{Zn}_{77.0} \mathrm{Pt}_{23.0}$ & - & - & - & - & $\mathrm{md}$ & + & + & + & + & $\mathrm{md}$ & + & + & $\mathrm{m}$ & $\mathrm{m}$ & $\mathrm{m}$ & - & + & + & + & + & - & - & - \\
\hline $\mathrm{Zn}_{80.0} \mathrm{Pt}_{20.0}$ & - & - & - & - & + & + & $\mathrm{m}$ & + & $\mathrm{d}$ & + & + & + & + & + & + & + & $\mathrm{ms}$ & + & + & + & - & - & - \\
\hline $\mathrm{Zn}_{78.5} \mathrm{Pd}_{14.0} \mathrm{Al}_{7.5}$ & - & - & - & - & + & + & + & + & $\mathrm{m}$ & + & + & - & + & + & + & + & + & + & + & $\mathrm{m}$ & - & - & - \\
\hline $\mathrm{Cu}_{73.9} \mathrm{Sn}_{23.2} \mathrm{Ni}_{2.9}$ & - & - & - & - & + & + & + & + & + & + & d & + & + & + & + & + & $\mathrm{d}$ & + & + & + & - & - & - \\
\hline $\mathrm{Cu}_{78.6} \mathrm{Sn}_{21.4}$ & - & - & - & - & + & + & + & + & + & + & $\mathrm{d}$ & + & + & + & + & + & + & + & + & + & - & - & - \\
\hline $\mathrm{Cu}_{79.8} \mathrm{Sn}_{20.2}$ & - & - & - & - & + & + & + & + & + & + & + & $\mathrm{m}$ & $\mathrm{m}$ & + & + & + & + & + & + & $\mathrm{m}$ & - & - & - \\
\hline \multicolumn{24}{|c|}{ Subtype I/II (3 structures) } \\
\hline theoretical & + & + & + & + & + & + & + & + & + & + & d & + & + & $\mathrm{d}$ & + & + & + & + & + & + & - & $\mathrm{d}$ & - \\
\hline $\mathrm{Zn}_{89.1} \mathrm{Ir}_{10.9}$ & - & $\mathrm{d}$ & - & - & $\mathrm{m}$ & + & + & + & + & + & $\mathrm{d}$ & - & + & d & $\mathrm{m}$ & + & + & + & + & + & - & d & - \\
\hline $\mathrm{Zn}_{90.5} \mathrm{Ir}_{9.5}$ & - & $\mathrm{d}$ & $\mathrm{d}$ & - & $\mathrm{m}$ & + & + & + & + & ds & $\mathrm{d}$ & - & + & $\mathrm{d}$ & $\mathrm{m}$ & + & + & + & $\mathrm{s}$ & + & - & $\mathrm{d}$ & - \\
\hline $\mathrm{Zn}_{91.1} \mathrm{Ir}_{8.9}$ & - & $\mathrm{d}$ & d & - & $\mathrm{m}$ & + & + & + & + & ds & $\mathrm{d}$ & - & + & d & $\mathrm{m}$ & + & + & + & + & + & - & d & - \\
\hline \multicolumn{24}{|c|}{ Subtype II (12 structures) } \\
\hline theoretical & + & + & + & + & + & + & + & + & + & + & - & + & + & - & + & + & + & + & + & + & - & + & - \\
\hline $\mathrm{Na}_{86.3} \mathrm{Tl}_{13.7}$ & - & - & - & - & + & + & + & + & + & + & - & - & d & - & + & + & + & + & + & + & - & + & - \\
\hline $\mathrm{Sc}_{86.3} \mathrm{Os}_{13.7}$ & - & - & - & - & + & + & + & + & + & + & - & - & + & - & + & + & + & + & + & + & - & + & - \\
\hline $\mathrm{Mg}_{87.3} \mathrm{Ru}_{12.7}$ & - & - & - & - & + & + & + & $\mathrm{m}$ & + & $\mathrm{m}$ & - & - & + & - & + & + & + & + & + & + & - & + & - \\
\hline $\mathrm{Mg}_{86.3} \mathrm{Rh}_{13.7}$ & - & - & - & - & + & + & + & + & + & + & - & - & + & - & + & + & + & + & + & + & - & + & - \\
\hline $\mathrm{Mg}_{87.9} \operatorname{Ir}_{12.1}$ & - & + & - & - & + & + & + & + & + & - & - & - & + & - & + & + & + & + & + & + & - & + & - \\
\hline $\mathrm{Mg}_{85.9} \mathrm{Pd}_{14.1}$ & - & d & - & - & + & $\mathrm{m}$ & + & + & + & - & - & - & + & - & + & + & + & + & + & + & - & + & - \\
\hline $\mathrm{Mg}_{86.3} \operatorname{Ir}_{13.7}$ & - & - & - & - & + & + & + & + & + & + & - & - & + & - & + & + & + & + & + & + & - & + & - \\
\hline $\mathrm{Zn}_{95.3} \mathrm{Mo}_{4.7}$ & $\mathrm{~d}$ & + & $\mathrm{m}$ & - & + & + & + & + & $\mathrm{d}$ & d & - & - & + & - & $\mathrm{d}$ & + & + & + & + & + & - & + & - \\
\hline $\mathrm{Al}_{65.3} \mathrm{Cu}_{18.1} \mathrm{Cr}_{16.6}$ & - & $\mathrm{d}$ & - & - & + & + & + & $\mathrm{m}$ & $\mathrm{m}$ & + & - & - & + & - & + & $\mathrm{m}$ & + & + & + & $\mathrm{m}$ & - & $\mathrm{d}$ & - \\
\hline $\mathrm{Zn}_{86.6} \mathrm{Fe}_{6.7} \mathrm{Ni}_{6.7}$ & - & + & d & - & + & + & $\mathrm{m}$ & + & + & mds & - & - & + & - & $\mathrm{m}$ & + & + & + & + & + & - & + & - \\
\hline $\mathrm{Zn}_{78.4} \mathrm{Fe}_{21.6}$ & - & - & - & - & $\mathrm{m}$ & + & + & $\mathrm{m}$ & $\mathrm{m}$ & + & - & - & + & - & + & + & + & + & + & + & - & + & - \\
\hline $\mathrm{In}_{54.5} \mathrm{Pd}_{29.3} \mathrm{Ce}_{16.3}$ & - & + & - & - & 2 & + & + & + & + & + & - & + & + & - & + & + & + & + & $96 i$ & + & - & $\mathrm{s}$ & - \\
\hline $\mathrm{Zn}_{67.5} \mathrm{Ce}_{16.7} \mathrm{Mg}_{15.8}$ & - & + & + & - & + & + & + & + & + & + & - & + & + & - & + & + & + & + & $96 i$ & + & - & + & - \\
\hline \multicolumn{24}{|c|}{ Subtype III (3 structures) } \\
\hline theoretical & + & + & + & + & + & + & + & + & + & + & - & - & + & - & + & - & + & + & + & + & - & + & + \\
\hline $\mathrm{Mg}_{83.6} \mathrm{Gd}_{16.4}$ & + & + & + & + & + & + & + & + & + & $\mathrm{d}$ & - & - & + & - & $\mathrm{d}$ & - & + & $\mathrm{m}$ & + & + & - & + & + \\
\hline $\mathrm{Cd}_{80.4} \mathrm{Sm}_{19.6}$ & + & + & + & + & + & + & + & + & + & + & - & - & + & - & + & - & + & + & + & + & - & + & + \\
\hline $\mathrm{Al}_{63.6} \mathrm{Ta}_{36.4}$ & - & + & + & + & + & + & + & + & + & $\mathrm{s}$ & - & - & + & - & $\mathrm{s}$ & - & + & $\mathrm{s}$ & s & $\mathrm{sm}$ & - & s & + \\
\hline $\mathrm{Mg}_{82.4} \mathrm{Y}_{9.0} \mathrm{Ce}_{8.6}$ & + & $\mathrm{m}$ & $\mathrm{m}$ & + & + & + & + & $\mathrm{m}$ & + & $\mathrm{d}$ & - & - & + & - & $\mathrm{d}$ & - & + & $\mathrm{m}$ & + & + & - & + & + \\
\hline $\mathrm{Na}_{49.2} \mathrm{Ba}_{28.8} \mathrm{Li}_{22.0}$ & - & + & - & + & + & + & + & + & + & + & - & - & + & - & + & - & + & 2 & + & + & - & + & + \\
\hline \multicolumn{24}{|c|}{ Subtype IV (1 structure) } \\
\hline theoretical & + & + & + & + & + & + & + & + & + & - & - & - & + & - & - & - & + & + & + & + & + & + & + \\
\hline $\mathrm{Na}_{49.1} \mathrm{Sn}_{26.3} \operatorname{In}_{24.6}$ & + & + & + & + & + & + & + & + & + & - & - & - & + & - & - & - & + & + & + & + & + & $\mathrm{m}$ & + \\
\hline \multicolumn{24}{|c|}{ Subtype V (0 structures) } \\
\hline $\begin{array}{l}\text { theoretical } \\
\text { and site } 48 h 8\end{array}$ & + & + & + & + & + & + & + & + & - & - & - & - & - & - & - & - & + & + & + & + & + & + & + \\
\hline
\end{tabular}


Table 25

Site occupations of all discussed complex intermetallic structures in space group $F \overline{4} 3 m$. The atomic sorts, occupying the respective sites, are given. In the case of mixed occupations, the first letters of all featured elements are given (alternatively the second letters in case of ambiguities).

\begin{tabular}{|c|c|c|c|c|c|c|c|c|c|c|c|c|c|c|c|c|c|c|c|c|c|c|c|}
\hline \multirow[b]{2}{*}{ Structure } & \multicolumn{4}{|c|}{4} & \multicolumn{8}{|c|}{$16 \mathrm{e}$} & \multicolumn{2}{|c|}{$24 f$} & \multicolumn{2}{|c|}{$24 g$} & \multicolumn{7}{|c|}{$48 h$} \\
\hline & $a$ & $b$ & $c$ & $d$ & 1 & 2 & 3 & 4 & 5 & 6 & 7 & 8 & 1 & 2 & 1 & 2 & 1 & 2 & 3 & 4 & 5 & 6 & 7 \\
\hline \multicolumn{24}{|c|}{ Subtype I (10 structures) } \\
\hline theoretical & + & + & + & + & + & + & + & + & + & + & + & + & + & + & + & + & + & + & + & + & - & - & - \\
\hline $\mathrm{Li}_{64.3} \operatorname{In}_{26.5} \mathrm{Ag}_{9.2}$ & $\mathrm{Li}$ & L/A & $\mathrm{Li}$ & $\mathrm{A} / \mathrm{L}$ & $\mathrm{Ag}$ & In & $\mathrm{Li}$ & $\mathrm{Li}$ & L/A & $\mathrm{Li}$ & $\mathrm{Li}$ & $\mathrm{I} / \mathrm{L}$ & In & $\mathrm{Li}$ & $\mathrm{A} / \mathrm{L}$ & $\mathrm{Li}$ & $\mathrm{Li}$ & $\mathrm{Li}$ & $\mathrm{I} / \mathrm{L}$ & $\mathrm{I} / \mathrm{L}$ & - & - & - \\
\hline $\mathrm{Li}_{81.0} \mathrm{~Pb}_{19.0}$ & $\mathrm{Li}$ & - & - & - & $\mathrm{Pb}$ & $\mathrm{Li}$ & $\mathrm{Li}$ & $\mathrm{Pb}$ & $\mathrm{Li}$ & $\mathrm{Li}$ & $\mathrm{Li}$ & $\mathrm{Li}$ & $\mathrm{Li}$ & $\mathrm{Pb}$ & $\mathrm{Li}$ & $\mathrm{Pb}$ & $\mathrm{Li}$ & $\mathrm{Li}$ & $\mathrm{Li}$ & $\mathrm{Li}$ & - & - & - \\
\hline $\mathrm{Li}_{81.0} \mathrm{Sn}_{19.0}$ & $\mathrm{Li}$ & - & - & - & $\mathrm{Sn}$ & $\mathrm{Li}$ & $\mathrm{Li}$ & $\mathrm{Sn}$ & $\mathrm{Li}$ & $\mathrm{Li}$ & $\mathrm{Li}$ & $\mathrm{Li}$ & $\mathrm{Li}$ & $\mathrm{Sn}$ & $\mathrm{Li}$ & $\mathrm{Sn}$ & $\mathrm{Li}$ & $\mathrm{Li}$ & $\mathrm{Li}$ & $\mathrm{Li}$ & - & - & - \\
\hline $\mathrm{Li}_{81.0} \mathrm{Ge}_{19.0}$ & $\mathrm{Li}$ & - & - & - & $\mathrm{Ge}$ & $\mathrm{Li}$ & $\mathrm{Li}$ & $\mathrm{Ge}$ & $\mathrm{Li}$ & $\mathrm{Li}$ & $\mathrm{Li}$ & $\mathrm{Li}$ & $\mathrm{Li}$ & $\mathrm{Ge}$ & $\mathrm{Li}$ & $\mathrm{Ge}$ & $\mathrm{Li}$ & $\mathrm{Li}$ & $\mathrm{Li}$ & $\mathrm{Li}$ & - & - & - \\
\hline $\mathrm{Zn}_{80.9} \mathrm{Pd}_{14.5} \mathrm{Al}_{4.6}$ & - & - & - & - & $\mathrm{Pd}$ & $\mathrm{Pd}$ & $\mathrm{Zn}$ & $\mathrm{Pd}$ & Z/A & $\mathrm{Zn}$ & $\mathrm{Zn}$ & $\mathrm{A} / \mathrm{Z}$ & $\mathrm{Zn}$ & $\mathrm{Zn}$ & $\mathrm{Zn}$ & $\mathrm{P} / \mathrm{Z}$ & $\mathrm{Zn}$ & $\mathrm{Zn}$ & $\mathrm{Zn}$ & Z/A & - & - & - \\
\hline $\mathrm{Zn}_{82.7} \mathrm{Pt}_{17.3}$ & - & - & - & - & $\mathrm{Z} / \mathrm{P}$ & $\mathrm{Pt}$ & $\mathrm{Pt}$ & $\mathrm{Z} / \mathrm{P}$ & $\mathrm{Zn}$ & $\mathrm{Zn}$ & $\mathrm{Zn}$ & $\mathrm{Zn}$ & $\mathrm{P} / \mathrm{Z}$ & $\mathrm{Zn}$ & $\mathrm{Z} / \mathrm{P}$ & $\mathrm{Zn}$ & $\mathrm{Zn}$ & $\mathrm{Zn}$ & $\mathrm{Zn}$ & $\mathrm{Zn}$ & - & - & - \\
\hline $\mathrm{Zn}_{81.6} \mathrm{Pt}_{18.4}$ & - & - & - & - & $\mathrm{Zn}$ & $\mathrm{Pt}$ & $\mathrm{Pt}$ & $\mathrm{Z} / \mathrm{P}$ & $\mathrm{Zn}$ & $\mathrm{Zn}$ & $\mathrm{Zn}$ & $\mathrm{Zn}$ & $\mathrm{P} / \mathrm{Z}$ & $\mathrm{Zn}$ & $\mathrm{Z} / \mathrm{P}$ & $\mathrm{Zn}$ & $\mathrm{Zn}$ & $\mathrm{Zn}$ & $\mathrm{Zn}$ & $\mathrm{Zn}$ & - & - & - \\
\hline $\mathrm{Zn}_{78.7} \mathrm{Pd}_{16.0} \mathrm{Al}_{5.3}$ & - & - & - & - & $\mathrm{Pd}$ & $\mathrm{Pd}$ & $\mathrm{Zn}$ & $\mathrm{Pd}$ & Z/A & $\mathrm{Zn}$ & $\mathrm{Zn}$ & $\mathrm{Al}$ & $\mathrm{Zn}$ & $\mathrm{Zn}$ & $\mathrm{Zn}$ & $\mathrm{P} / \mathrm{Z}$ & $\mathrm{Zn}$ & $\mathrm{Zn}$ & $\mathrm{Zn}$ & $\mathrm{Z} / \mathrm{A}$ & - & - & - \\
\hline $\mathrm{Zn}_{77.0} \mathrm{Pt}_{23.0}$ & - & - & - & - & $\mathrm{Z} / \mathrm{P}$ & $\mathrm{Pt}$ & $\mathrm{Pt}$ & $\mathrm{Pt}$ & $\mathrm{Zn}$ & $\mathrm{Z} / \mathrm{P}$ & $\mathrm{Zn}$ & $\mathrm{Zn}$ & $\mathrm{P} / \mathrm{Z}$ & $\mathrm{Z} / \mathrm{P}$ & $\mathrm{P} / \mathrm{Z}$ & - & $\mathrm{Zn}$ & $\mathrm{Zn}$ & $\mathrm{Zn}$ & $\mathrm{Zn}$ & - & - & - \\
\hline $\mathrm{Zn}_{80.0} \mathrm{Pt}_{20.0}$ & - & - & - & - & $\mathrm{Pt}$ & $\mathrm{Pt}$ & $\mathrm{Z} / \mathrm{P}$ & $\mathrm{Zn}$ & $\mathrm{Zn}$ & $\mathrm{Zn}$ & $\mathrm{Zn}$ & $\mathrm{Zn}$ & $\mathrm{Pt}$ & $\mathrm{Zn}$ & $\mathrm{Zn}$ & $\mathrm{Pt}$ & $\mathrm{Zn}$ & $\mathrm{Zn}$ & $\mathrm{Zn}$ & $\mathrm{Zn}$ & - & - & - \\
\hline $\mathrm{Zn}_{78.5} \mathrm{Pd}_{14.0} \mathrm{Al}_{7.5}$ & - & - & - & - & $\mathrm{Zn}$ & $\mathrm{Pd}$ & $\mathrm{Al}$ & $\mathrm{Pd}$ & $\mathrm{Z} / \mathrm{A}$ & $\mathrm{Zn}$ & $\mathrm{Zn}$ & - & $\mathrm{Zn}$ & $\mathrm{Zn}$ & $\mathrm{Zn}$ & $\mathrm{Pd}$ & $\mathrm{Zn}$ & $\mathrm{Zn}$ & $\mathrm{Zn}$ & $\mathrm{Z} / \mathrm{A}$ & - & - & - \\
\hline $\mathrm{Cu}_{73.9} \mathrm{Sn}_{23.2} \mathrm{Ni}_{2.9}$ & - & - & - & - & $\mathrm{Cu}$ & Sn & $\mathrm{Cu}$ & $\mathrm{Cu}$ & $\mathrm{Cu}$ & $\mathrm{Cu}$ & $\mathrm{Cu}$ & $\mathrm{Cu}$ & $\mathrm{Cu}$ & $\mathrm{Cu}$ & $\mathrm{Sn}$ & $\mathrm{Cu}$ & $\mathrm{Cu}$ & $\mathrm{Cu}$ & $\mathrm{Cu}$ & $\mathrm{Sn}$ & - & - & - \\
\hline $\mathrm{Cu}_{78.6} \mathrm{Sn}_{21.4}$ & - & - & - & - & $\mathrm{Cu}$ & $\mathrm{Sn}$ & $\mathrm{Cu}$ & $\mathrm{Cu}$ & $\mathrm{Cu}$ & $\mathrm{Cu}$ & $\mathrm{Cu}$ & $\mathrm{Cu}$ & $\mathrm{Cu}$ & $\mathrm{Cu}$ & Sn & $\mathrm{Cu}$ & $\mathrm{Cu}$ & $\mathrm{Cu}$ & $\mathrm{Cu}$ & $\mathrm{Sn}$ & - & - & - \\
\hline $\mathrm{Cu}_{79.8} \mathrm{Sn}_{20.2}$ & - & - & - & - & $\mathrm{Cu}$ & $\mathrm{Sn}$ & $\mathrm{Cu}$ & $\mathrm{Cu}$ & $\mathrm{Cu}$ & $\mathrm{Cu}$ & $\mathrm{Cu}$ & $\mathrm{C} / \mathrm{S}$ & $\mathrm{C} / \mathrm{S}$ & $\mathrm{Cu}$ & $\mathrm{Sn}$ & $\mathrm{Cu}$ & $\mathrm{Cu}$ & $\mathrm{Cu}$ & $\mathrm{Cu}$ & $\mathrm{C} / \mathrm{S}$ & - & - & - \\
\hline \multicolumn{24}{|c|}{ Subtype I/II (3 structures) } \\
\hline theoretical & + & + & + & + & + & + & + & + & + & + & $\mathrm{d}$ & + & + & $\mathrm{d}$ & + & + & + & + & + & + & - & $\mathrm{d}$ & - \\
\hline $\mathrm{Zn}_{89.1} \mathrm{Ir}_{10.9}$ & - & $\mathrm{Zn}$ & - & - & $\mathrm{Z} / \mathrm{I}$ & $\mathrm{Zn}$ & Ir & Ir & $\mathrm{Zn}$ & $\mathrm{Zn}$ & $\mathrm{Zn}$ & 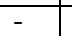 & $\mathrm{Zn}$ & $\mathrm{Zn}$ & Z/I & $\mathrm{Zn}$ & $\mathrm{Zn}$ & $\mathrm{Zn}$ & $\mathrm{Zn}$ & $\mathrm{Zn}$ & - & $\mathrm{Zn}$ & - \\
\hline $\mathrm{Zn}_{90.5} \mathrm{Ir}_{9.5}$ & - & $\mathrm{Zn}$ & Ir & - & $\mathrm{Z} / \mathrm{I}$ & $\mathrm{Zn}$ & Ir & Ir & $\mathrm{Zn}$ & $\mathrm{Zn}$ & $\mathrm{Zn}$ & - & $\mathrm{Zn}$ & $\mathrm{Zn}$ & $\mathrm{Z} / \mathrm{I}$ & $\mathrm{Zn}$ & $\mathrm{Zn}$ & $\mathrm{Zn}$ & $\mathrm{Zn}$ & $\mathrm{Zn}$ & - & $\mathrm{Zn}$ & - \\
\hline $\mathrm{Zn}_{91.1} \mathrm{Ir}_{8.9}$ & - & $\mathrm{Zn}$ & Ir & - & $\mathrm{Z} / \mathrm{I}$ & $\mathrm{Zn}$ & Ir & Ir & $\mathrm{Zn}$ & $\mathrm{Zn}$ & $\mathrm{Zn}$ & - & $\mathrm{Zn}$ & $\mathrm{Zn}$ & $\mathrm{Z} / \mathrm{I}$ & $\mathrm{Zn}$ & $\mathrm{Zn}$ & $\mathrm{Zn}$ & $\mathrm{Zn}$ & $\mathrm{Zn}$ & - & $\mathrm{Zn}$ & - \\
\hline \multicolumn{24}{|c|}{ Subtype II (12 structures) } \\
\hline theoretical & + & + & + & + & + & + & + & + & + & + & - & + & + & - & + & + & + & + & + & + & - & + & - \\
\hline $\mathrm{Na}_{86.3} \mathrm{Tl}_{13.7}$ & - & - & - & - & $\mathrm{Na}$ & $\mathrm{Na}$ & $\mathrm{Tl}$ & $\mathrm{Tl}$ & $\mathrm{Na}$ & $\mathrm{Na}$ & - & - & $\mathrm{Na}$ & - & $\mathrm{Tl}$ & $\mathrm{Na}$ & $\mathrm{Na}$ & $\mathrm{Na}$ & $\mathrm{Na}$ & $\mathrm{Na}$ & - & $\mathrm{Na}$ & - \\
\hline $\mathrm{Sc}_{86.3} \mathrm{Os}_{13.7}$ & - & - & - & - & $\mathrm{Sc}$ & $\mathrm{Sc}$ & Os & Os & $\mathrm{Sc}$ & Sc & - & - & $\mathrm{Sc}$ & - & Os & Sc & $\mathrm{Sc}$ & $\mathrm{Sc}$ & $\mathrm{Sc}$ & Sc & - & Sc & - \\
\hline $\mathrm{Mg}_{87.3} \mathrm{Ru}_{12.7}$ & - & - & - & - & $\mathrm{Mg}$ & $\mathrm{Mg}$ & $\mathrm{Ru}$ & $\mathrm{R} / \mathrm{M}$ & $\mathrm{Mg}$ & $\mathrm{M} / \mathrm{R}$ & - & - & $\mathrm{Mg}$ & - & $\mathrm{Ru}$ & $\mathrm{Mg}$ & $\mathrm{Mg}$ & $\mathrm{Mg}$ & $\mathrm{Mg}$ & $\mathrm{Mg}$ & - & $\mathrm{Mg}$ & - \\
\hline $\mathrm{Mg}_{86.3} \mathrm{Rh}_{13.7}$ & - & - & - & - & $\mathrm{Mg}$ & $\mathrm{Mg}$ & $\mathrm{Rh}$ & $\mathrm{Rh}$ & $\mathrm{Mg}$ & $\mathrm{Mg}$ & - & - & $\mathrm{Mg}$ & - & $\mathrm{Rh}$ & $\mathrm{Mg}$ & $\mathrm{Mg}$ & $\mathrm{Mg}$ & $\mathrm{Mg}$ & $\mathrm{Mg}$ & - & $\mathrm{Mg}$ & - \\
\hline $\mathrm{Mg}_{87.9} \operatorname{Ir}_{12.1}$ & - & $\mathrm{Mg}$ & - & - & $\mathrm{Ir}$ & $\mathrm{Mg}$ & Ir & Ir & $\mathrm{Mg}$ & - & - & - & $\mathrm{Mg}$ & - & $\mathrm{Mg}$ & $\mathrm{Mg}$ & $\mathrm{Mg}$ & $\mathrm{Mg}$ & $\mathrm{Mg}$ & $\mathrm{Mg}$ & - & $\mathrm{Mg}$ & - \\
\hline $\mathrm{Mg}_{85.9} \mathrm{Pd}_{14.1}$ & - & $\mathrm{Mg}$ & - & - & $\mathrm{Pd}$ & $\mathrm{M} / \mathrm{P}$ & $\mathrm{Pd}$ & $\mathrm{Pd}$ & $\mathrm{Mg}$ & - & - & - & $\mathrm{Mg}$ & - & $\mathrm{Mg}$ & $\mathrm{Mg}$ & $\mathrm{Mg}$ & $\mathrm{Mg}$ & $\mathrm{Mg}$ & $\mathrm{Mg}$ & - & $\mathrm{Mg}$ & - \\
\hline $\operatorname{Mg}_{86.3} \operatorname{Ir}_{13.7}$ & - & - & - & - & $\mathrm{Mg}$ & $\mathrm{Mg}$ & $\mathrm{Ir}$ & $\mathrm{Ir}$ & $\mathrm{Mg}$ & $\mathrm{Mg}$ & - & - & $\mathrm{Mg}$ & - & $\mathrm{Ir}$ & $\mathrm{Mg}$ & $\mathrm{Mg}$ & $\mathrm{Mg}$ & $\mathrm{Mg}$ & $\mathrm{Mg}$ & - & $\mathrm{Mg}$ & - \\
\hline $\mathrm{Zn}_{95.3} \mathrm{Mo}_{4.7}$ & $\mathrm{Zn}$ & $\mathrm{Zn}$ & $\mathrm{M} / \mathrm{Z}$ & - & $\mathrm{Zn}$ & $\mathrm{Zn}$ & Mo & $\mathrm{Zn}$ & $\mathrm{Zn}$ & $\mathrm{Zn}$ & - & - & $\mathrm{Zn}$ & - & $\mathrm{Zn}$ & $\mathrm{Zn}$ & $\mathrm{Zn}$ & $\mathrm{Zn}$ & $\mathrm{Zn}$ & $\mathrm{Zn}$ & - & $\mathrm{Zn}$ & - \\
\hline $\mathrm{Al}_{65.3 .3} \mathrm{Cu}_{18.1} \mathrm{Cr}_{16.6}$ & - & $\mathrm{Cu}$ & - & - & $\mathrm{Cr}$ & $\mathrm{Al}$ & $\mathrm{Cr}$ & $\mathrm{r} / \mathrm{u}$ & $\mathrm{A} / \mathrm{u}$ & $\mathrm{Al}$ & - & - & $\mathrm{Al}$ & - & $\mathrm{Cr}$ & $\mathrm{A} / \mathrm{u}$ & $\mathrm{Al}$ & $\mathrm{Al}$ & $\mathrm{Al}$ & $\mathrm{A} / \mathrm{u}$ & - & $\mathrm{Cu}$ & - \\
\hline $\mathrm{Zn}_{86.6} \mathrm{Fe}_{6.7} \mathrm{Ni}_{6.7}$ & - & $\mathrm{Zn}$ & $\mathrm{F} / \mathrm{N}$ & - & $\mathrm{Zn}$ & $\mathrm{Zn}$ & $\mathrm{F} / \mathrm{N}$ & $\mathrm{Zn}$ & $\mathrm{Zn}$ & $\mathrm{F} / \mathrm{N}$ & - & - & $\mathrm{Zn}$ & - & $\mathrm{F} / \mathrm{N}$ & $\mathrm{Zn}$ & $\mathrm{Zn}$ & $\mathrm{Zn}$ & $\mathrm{Zn}$ & $\mathrm{Zn}$ & - & $\mathrm{Zn}$ & - \\
\hline $\mathrm{Zn}_{78.4} \mathrm{Fe}_{21.6}$ & - & - & - & - & $F / Z$ & $\mathrm{Zn}$ & $\mathrm{Fe}$ & $\mathrm{F} / \mathrm{Z}$ & $F / Z$ & $\mathrm{Fe}$ & - & - & $\mathrm{Zn}$ & - & $\mathrm{Fe}$ & $\mathrm{Zn}$ & $\mathrm{Zn}$ & $\mathrm{Zn}$ & $\mathrm{Zn}$ & $\mathrm{Zn}$ & - & $\mathrm{Zn}$ & - \\
\hline $\mathrm{In}_{54.5} \mathrm{Pd}_{29.3} \mathrm{Ce}_{16.3}$ & - & In & - & - & $P+I$ & $\mathrm{Ce}$ & $\mathrm{Ce}$ & $\mathrm{Pd}$ & In & $\mathrm{Pd}$ & - & In & $\mathrm{Ce}$ & - & $\mathrm{Ce}$ & In & In & In & $P d$ & In & - & In & - \\
\hline $\mathrm{Zn}_{67.5} \mathrm{Ce}_{16.7} \mathrm{Mg}_{15.8}$ & - & $\mathrm{Mg}$ & $\mathrm{Zn}$ & - & $\mathrm{Zn}$ & $\mathrm{Ce}$ & $\mathrm{Ce}$ & $\mathrm{Zn}$ & $\mathrm{Zn}$ & $\mathrm{Zn}$ & - & $\mathrm{Zn}$ & $\mathrm{Ce}$ & - & $\mathrm{Ce}$ & $\mathrm{Mg}$ & $\mathrm{Mg}$ & $\mathrm{Zn}$ & $\mathrm{Zn}$ & $\mathrm{Zn}$ & - & $\mathrm{Zn}$ & - \\
\hline \multicolumn{24}{|c|}{ Subtype III (3 structures) } \\
\hline theoretical & + & + & + & + & + & + & + & + & + & + & - & - & + & - & + & - & + & + & + & + & - & + & + \\
\hline $\mathrm{Mg}_{83.6} \mathrm{Gd}_{16.4}$ & $\mathrm{Mg}$ & Gd & $\mathrm{Gd}$ & $\mathrm{Mg}$ & $\mathrm{Mg}$ & $\mathrm{Mg}$ & $\mathrm{Gd}$ & $\mathrm{Gd}$ & $\mathrm{Mg}$ & $\mathrm{Mg}$ & - & - & $\mathrm{Mg}$ & - & $\mathrm{Mg}$ & - & $\mathrm{Mg}$ & G/M & $\mathrm{Mg}$ & $\mathrm{Mg}$ & - & $\mathrm{Mg}$ & $\mathrm{Mg}$ \\
\hline $\mathrm{Cd}_{80.4} \mathrm{Sm}_{19.6}$ & $\mathrm{Cd}$ & Sm & $\mathrm{Sm}$ & $\mathrm{Cd}$ & $\mathrm{Cd}$ & $\mathrm{Cd}$ & Sm & $\mathrm{Sm}$ & $\mathrm{Cd}$ & $\mathrm{Cd}$ & - & - & $\mathrm{Cd}$ & - & $\mathrm{Cd}$ & - & $\mathrm{Cd}$ & $\mathrm{Sm}$ & $\mathrm{Cd}$ & $\mathrm{Cd}$ & - & $\mathrm{Cd}$ & $\mathrm{Cd}$ \\
\hline $\mathrm{Al}_{63.6} \mathrm{Ta}_{36.4}$ & - & $\mathrm{Ta}$ & $\mathrm{Ta}$ & $\mathrm{Ta}$ & $\mathrm{Al}$ & $\mathrm{Ta}$ & $\mathrm{Ta}$ & $\mathrm{Ta}$ & $\mathrm{Al}$ & $\mathrm{Al}$ & - & - & $\mathrm{Al}$ & - & $\mathrm{Al}$ & - & $\mathrm{Ta}$ & $\mathrm{Al}$ & $\mathrm{Ta}$ & $\mathrm{A} / \mathrm{T}$ & - & $\mathrm{Al}$ & $\mathrm{Al}$ \\
\hline $\mathrm{Mg}_{82.4} \mathrm{Y}_{9.0} \mathrm{Ce}_{8.6}$ & $\mathrm{Mg}$ & $\mathrm{C} / \mathrm{Y}$ & $\mathrm{C} / \mathrm{Y}$ & $\mathrm{Mg}$ & $\mathrm{Mg}$ & $\mathrm{Mg}$ & $\mathrm{Y}$ & $\mathrm{C} / \mathrm{Y}$ & $\mathrm{Mg}$ & $\mathrm{Mg}$ & - & - & $\mathrm{Mg}$ & - & $\mathrm{Mg}$ & - & $\mathrm{Mg}$ & $C Y M$ & $1 \mathrm{Mg}$ & $\mathrm{Mg}$ & - & $\mathrm{Mg}$ & $\mathrm{Mg}$ \\
\hline $\mathrm{Na}_{49.2} \mathrm{Ba}_{28.8} \mathrm{Li}_{22.0}$ & - & $\mathrm{Ba}$ & - & $\mathrm{Ba}$ & $\mathrm{Li}$ & $\mathrm{Ba}$ & $\mathrm{Ba}$ & $\mathrm{Ba}$ & $\mathrm{Na}$ & $\mathrm{Li}$ & - & - & $\mathrm{Na}$ & - & $\mathrm{Li}$ & - & $\mathrm{Ba}$ & $N+L$ & $\mathrm{Ba}$ & $\mathrm{Na}$ & - & $\mathrm{Na}$ & $\mathrm{Na}$ \\
\hline \multicolumn{24}{|c|}{ Subtype IV (1 structure) } \\
\hline theoretical & + & + & + & + & + & + & + & + & + & - & - & - & + & - & - & - & + & + & + & + & + & + & + \\
\hline $\mathrm{Na}_{49.1} \mathrm{Sn}_{26.3} \mathrm{In}_{24.6}$ & $\mathrm{Na}$ & $\mathrm{Na}$ & $\mathrm{Na}$ & $\mathrm{Na}$ & $\mathrm{Na}$ & $\mathrm{Na}$ & $\mathrm{Na}$ & $\mathrm{Na}$ & $\mathrm{Sn}$ & - & - & - & In & - & - & - & $\mathrm{Na}$ & $\mathrm{Na}$ & $\mathrm{Na}$ & $\mathrm{Sn}$ & Sn & $\mathrm{I} / \mathrm{S}$ & In \\
\hline
\end{tabular}

\section{Table 26}

Site occupations of all discussed complex intermetallic structures in space group $F d \overline{3} m$. A + denotes that a site is occupied, a - that it is empty. Sites labelled with different letters are occupied the following modifications: $\mathrm{m}$ - mixed occupation, $\mathrm{d}$ - deficient occupation. Also given are - in the right half of the table - the atomic sorts, occupying the respective sites. The additional site found in $\mathrm{Zn}_{61.0} \mathrm{Ca}_{35.6} \mathrm{Ni}_{3.4}-16 d-$ is fully occupied by Ni.

\begin{tabular}{|c|c|c|c|c|c|c|c|c|c|c|c|c|c|c|c|c|}
\hline \multirow[b]{2}{*}{ Structure } & \multicolumn{2}{|c|}{8} & \multicolumn{2}{|c|}{$32 \mathrm{e}$} & \multicolumn{4}{|c|}{$96 g$} & \multicolumn{2}{|c|}{8} & \multicolumn{2}{|c|}{$32 \mathrm{e}$} & \multicolumn{4}{|c|}{$96 g$} \\
\hline & $a$ & $b$ & 1 & 2 & 1 & 2 & 3 & 4 & $a$ & $b$ & 1 & 2 & 1 & 2 & 3 & 4 \\
\hline $\operatorname{In}_{70.7} \mathrm{~K}_{29.3}$ & + & + & + & + & + & + & + & + & In & $\mathrm{K}$ & In & $\mathrm{K}$ & $\mathrm{K}$ & In & In & In \\
\hline $\mathrm{Ga}_{50.0} \mathrm{Na}_{29.3} \mathrm{In}_{20.7}$ & + & + & + & + & + & + & + & + & $\mathrm{Ga}$ & $\mathrm{Na}$ & $\mathrm{Ga}$ & $\mathrm{Na}$ & $\mathrm{Na}$ & In & $\mathrm{Ga}$ & $\mathrm{Ga}$ \\
\hline $\mathrm{Ga}_{48.9} \mathrm{Na}_{30.4} \mathrm{Cd}_{20.7}$ & + & $\mathrm{d}$ & $\mathrm{m}$ & + & + & $\mathrm{m}$ & + & + & $\mathrm{Na}$ & $\mathrm{Na}$ & $\mathrm{Cd} / \mathrm{Ga}$ & $\mathrm{Na}$ & $\mathrm{Na}$ & $\mathrm{Cd} / \mathrm{Ga}$ & $\mathrm{Ga}$ & $\mathrm{Ga}$ \\
\hline $\mathrm{Ga}_{63.0} \mathrm{Na}_{31.0} \mathrm{Ag}_{6.0}$ & + & + & $\mathrm{m}$ & + & + & $\mathrm{m}$ & + & + & $\mathrm{Na}$ & $\mathrm{Na}$ & $\mathrm{Ag} / \mathrm{Ga}$ & $\mathrm{Na}$ & $\mathrm{Na}$ & $\mathrm{Ag} / \mathrm{Ga}$ & $\mathrm{Ga}$ & $\mathrm{Ga}$ \\
\hline $\mathrm{Ga}_{53.4} \mathrm{Li}_{31.0} \mathrm{Cu}_{8.6} \operatorname{In}_{6.9}$ & + & + & + & + & + & + & + & $\mathrm{m}$ & $\mathrm{Li}$ & $\mathrm{Li}$ & In & $\mathrm{Li}$ & $\mathrm{Li}$ & $\mathrm{Ga}$ & $\mathrm{Ga}$ & $\mathrm{Ga} / \mathrm{Cu}$ \\
\hline $\mathrm{Ga}_{47.5} \mathrm{Mg}_{31.2} \mathrm{Cu}_{21.3}$ & $\mathrm{~d}$ & + & $\mathrm{d}$ & + & + & + & + & + & $\mathrm{Mg}$ & $\mathrm{Mg}$ & $\mathrm{Ga}$ & $\mathrm{Mg}$ & $\mathrm{Mg}$ & $\mathrm{Ga}$ & $\mathrm{Cu}$ & $\mathrm{Ga}$ \\
\hline $\mathrm{Zn}_{61.0} \mathrm{Ca}_{35.6} \mathrm{Ni}_{3.4}$ & - & + & + & + & + & + & + & + & & $\mathrm{Ca}$ & $\mathrm{Ca}$ & $\mathrm{Ca}$ & $\mathrm{Ca}$ & $\mathrm{Zn}$ & $\mathrm{Zn}$ & $\mathrm{Zn}$ \\
\hline
\end{tabular}


5. Coordination shells around low-symmetry sites in $\mathbf{A l}_{63.6} \mathbf{T a}_{36.4}$
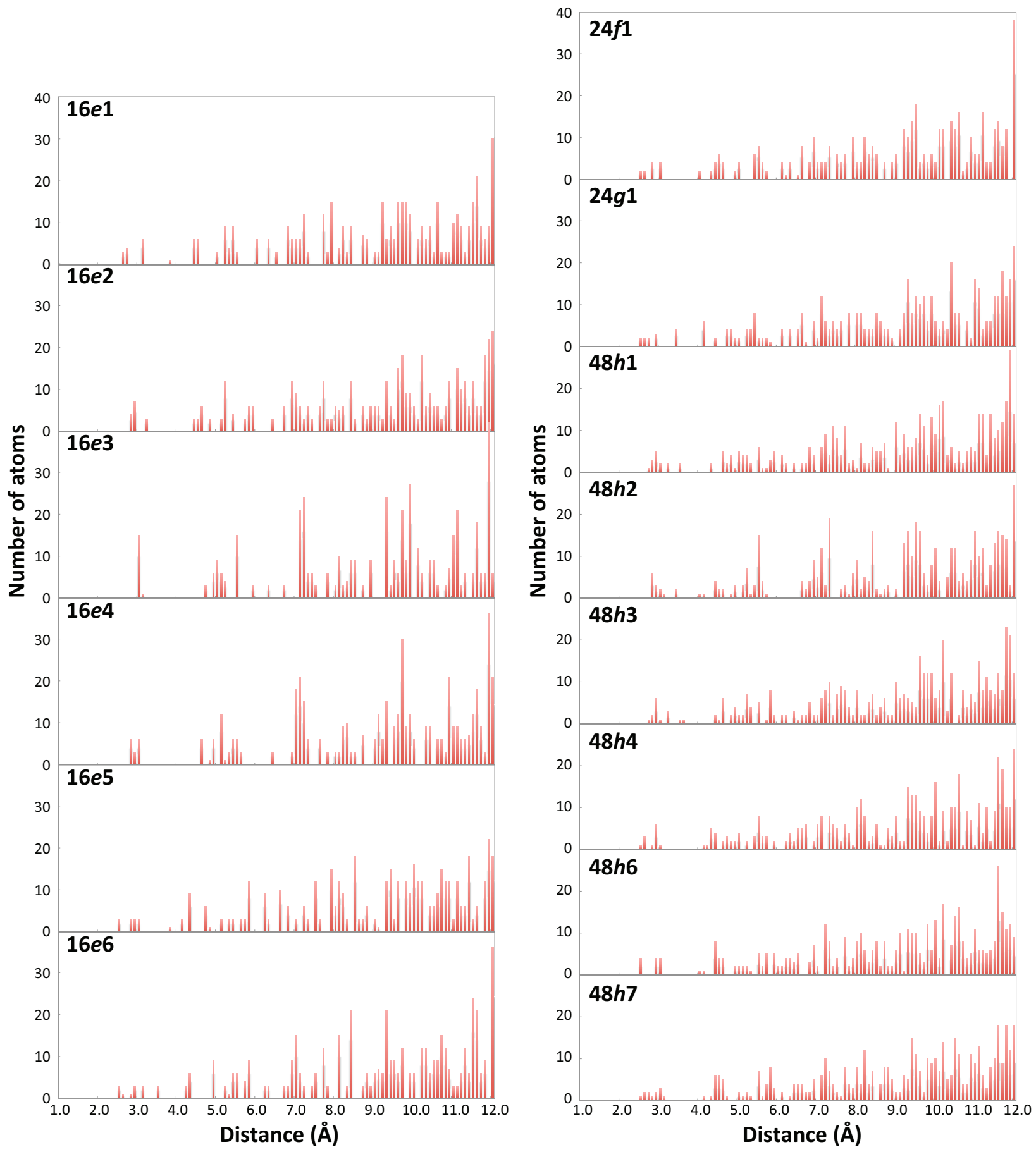

Figure 1

Frequency of interatomic distances of atoms in the environment of the lower-symmetry atomic sites in $\mathrm{Al}_{63.6} \mathrm{Ta}_{36.4}$. 


\section{6. (110) layers}
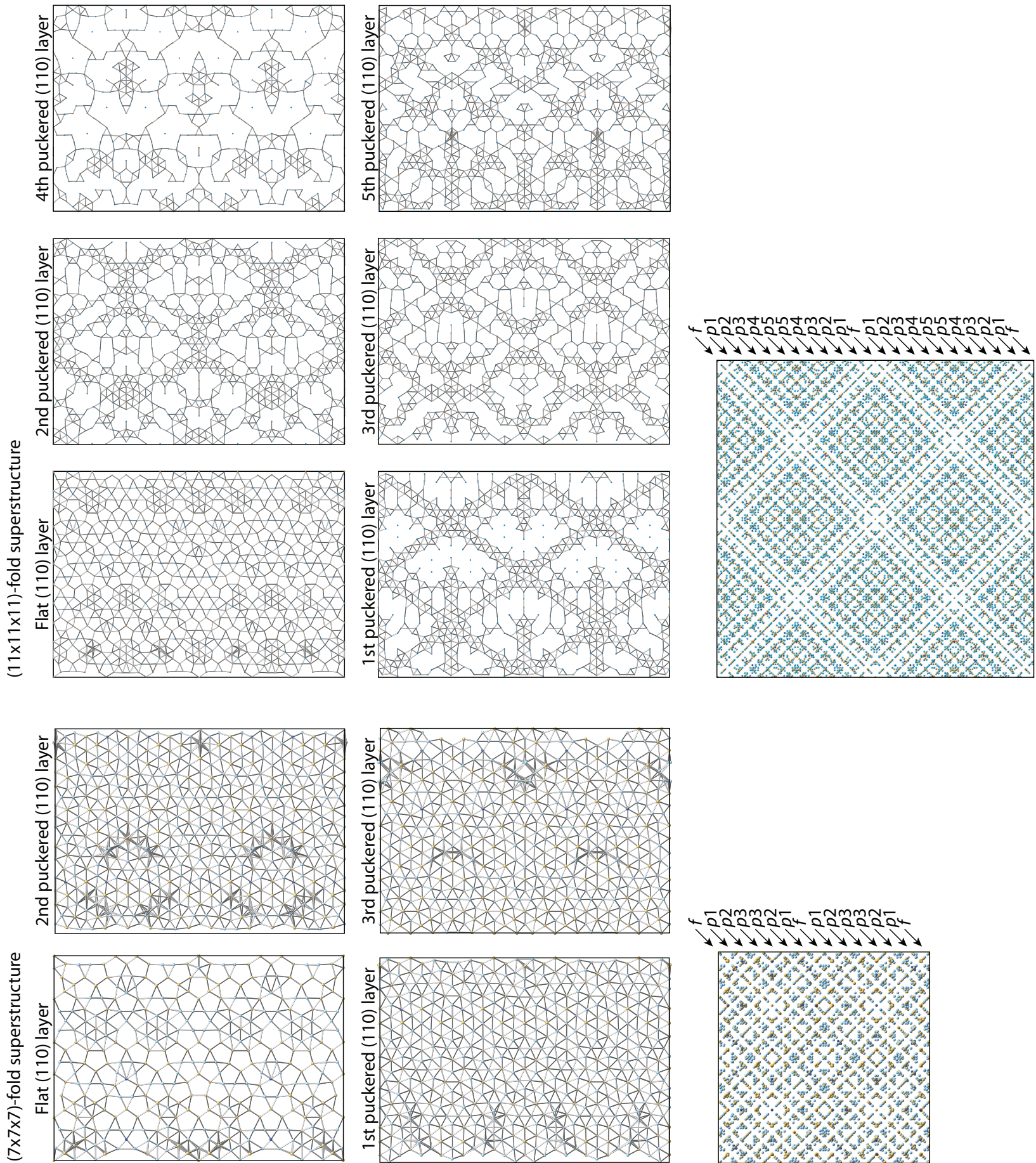

Figure 2

(110) layers of the two giant-unit-cell Al-Cu-Ta compounds (with $p=7$ and $p=11$ ), as well as their arrangement. 


\section{First-principles calculations}

\section{Table 27}

Details on the performed ab initio calculations for structures of space group $F \overline{4} 3 m$. Given are the number of calculated electrons per atom $(e /$ atom) and the number of atoms in the calculated unit cell (atoms/u.c.), both values given for each atomic sort in the order of content in the unit cell, as well as the idealization steps applied to partly disordered structures. The structures $\mathrm{Cu}_{73.9} \mathrm{Sn}_{23.2} \mathrm{Ni}_{2.9}$ and $\mathrm{Zn}_{86.6} \mathrm{Fe}_{6.7} \mathrm{Ni}_{6.7}$ had to be calculated in the variants $\mathrm{Cu}_{76.8} \mathrm{Sn}_{23.2}$ and $\mathrm{Zn}_{86.6} \mathrm{Fe}_{13.4}$, as well as $\mathrm{Zn}_{86.6} \mathrm{Ni}_{13.4}$, respectively, because the $\mathrm{Ni}$ sites had not been specified on structure determination.

\begin{tabular}{|c|c|c|c|}
\hline Structure & e/atom & atoms/u.c. & Applied idealization steps \\
\hline \multicolumn{4}{|c|}{ Subtype I (14 structures) } \\
\hline $\mathrm{Li}_{64.3} \operatorname{In}_{26.5} \mathrm{Ag}_{9.2}$ & $3,13,11$ & $59,38,11$ & $\begin{array}{l}\text { 4b: } 0.8 \mathrm{Li}, 0.2 \mathrm{Ag} \rightarrow 1 \mathrm{Li} ; 4 d: 0.82 \mathrm{Ag}, 0.18 \mathrm{Li} \rightarrow 1 \mathrm{Ag} ; 16 e 5: 0.57 \mathrm{Li}, 0.43 \mathrm{Ag} \rightarrow 1 \mathrm{Li} ; \\
\text { 16e8: } 0.77 \mathrm{In}, 0.23 \mathrm{Li} \rightarrow 1 \mathrm{In} ; 24 g 1: 0.54 \mathrm{Ag}, 0.46 \mathrm{Li} \rightarrow 1 \mathrm{Ag} ; 48 h 3: 0.54 \mathrm{In}, 0.46 \mathrm{Li} \rightarrow 1 \mathrm{In} ; \\
\text { 48h4: } 0.75 \mathrm{In}, 0.25 \mathrm{Li} \rightarrow 1 \mathrm{In}\end{array}$ \\
\hline $\mathrm{Li}_{81.0} \mathrm{~Pb}_{19.0}$ & 3,14 & 85,20 & - \\
\hline $\mathrm{Li}_{81.0} \mathrm{Sn}_{19.0}$ & 3,14 & 85,20 & - \\
\hline $\mathrm{Li}_{81.0} \mathrm{Ge}_{19.0}$ & 3,14 & 85,20 & $4 a: 0.75 \rightarrow 1 \mathrm{Li} ; 16 e 5: 0.75 / 0.25 \rightarrow 1 \mathrm{Li}$ \\
\hline $\mathrm{Zn}_{80.9} \mathrm{Pd}_{14.5} \mathrm{Al}_{4.6}$ & $12,16,3$ & $82,18,4$ & $\begin{array}{l}\text { 16e3: } 0.83 / 0.19 \mathrm{Zn} \rightarrow 1 \mathrm{Zn} ; 16 e 5: 0.94 \mathrm{Zn}, 0.05 \mathrm{Al} \rightarrow 1 \mathrm{Zn} ; 16 e 8: 0.78 \mathrm{Al}, 0.22 \mathrm{Zn} \rightarrow 1 \mathrm{Al} \\
\text { 24g2: } 0.52 \mathrm{Pd}, 0.48 \mathrm{Zn} \rightarrow 1 \mathrm{Pd} ; 48 h 4: 0.88 \mathrm{Zn}, 0.12 \mathrm{Al} \rightarrow 1 \mathrm{Zn}\end{array}$ \\
\hline $\mathrm{Zn}_{82.7} \mathrm{Pt}_{17.3}$ & 12,10 & 86,18 & $\begin{array}{l}16 e 1: 0.24 / 0.39 \mathrm{Zn}, 0.34 \mathrm{Pt} \rightarrow 1 \mathrm{Zn} ; 16 e 4: 0.61 \mathrm{Zn}, 0.39 \mathrm{Pt} \rightarrow 1 \mathrm{Zn} ; 16 e 6: 0.5 / 0.27 \rightarrow 1 \mathrm{Zn} \\
24 f 1: 0.51 \mathrm{Pt}, 0.49 \mathrm{Zn} \rightarrow 1 \mathrm{Pt} ; 24 f 2: 0.71 \rightarrow 1 \mathrm{Zn} ; 24 g 1: 0.53 \mathrm{Zn}, 0.47 \mathrm{Pt} \rightarrow 1 \mathrm{Zn} ; \\
24 g 2: 0.69 \rightarrow 1 \mathrm{Zn} ; 48 h 2: 0.91 \rightarrow 1 \mathrm{Zn}\end{array}$ \\
\hline $\mathrm{Zn}_{81.6} \mathrm{Pt}_{18.4}$ & 12,10 & 90,14 & $\begin{array}{l}\text { 16e }: 0.70 / 0.31 \rightarrow 1 \mathrm{Zn} ; 16 e 4: 0.86 / 0.14 \rightarrow 1 \mathrm{Zn} ; 16 e 6: 0.68 / 0.17 \rightarrow 1 \mathrm{Zn} ; \\
24 f 1: 0.85 \mathrm{Pt}, 0.15 \mathrm{Zn} \rightarrow 1 \mathrm{Pt} ; 24 g 1: 0.86 \mathrm{Pt}, 0.14 \mathrm{Zn} \rightarrow 1 \mathrm{Pt} ; 48 h 2: 0.90 \rightarrow 1 \mathrm{Zn}\end{array}$ \\
\hline $\mathrm{Zn}_{78.7} \mathrm{Pd}_{16.0} \mathrm{Al}_{5.3}$ & $12,16,3$ & $82,18,4$ & $\begin{array}{l}\text { 16e3: } 0.51 / 0.21 \rightarrow 1 \mathrm{Zn} ; 16 e 5: 0.78 \mathrm{Zn}, 0.22 \mathrm{Al} \rightarrow 1 \mathrm{Zn} ; 16 e 8: 0.74 \rightarrow 1 \mathrm{Al} \\
24 g 2: 0.71 \mathrm{Pd}, 0.29 \mathrm{Zn} \rightarrow 1 \mathrm{Pd} ; 48 h 4: 0.87 \mathrm{Zn}, 0.13 \mathrm{Al} \rightarrow 1 \mathrm{Zn}\end{array}$ \\
\hline $\mathrm{Zn}_{77.0} \mathrm{Pt}_{23.0}$ & 12,10 & 70,24 & $\begin{array}{l}16 e 1: 0.5 \mathrm{Zn}, 0.5 \mathrm{Pt} \rightarrow 1 \mathrm{Zn} ; 16 e 6: 0.92 \mathrm{Zn}, 0.18 \mathrm{Pt} \rightarrow 1 \mathrm{Zn} ; 24 f 1: 0.67 \mathrm{Pt}, 0.33 \mathrm{Zn} \rightarrow 1 \mathrm{Pt} \\
24 f 2: 0.92 \mathrm{Zn}, 0.18 \mathrm{Pt} \rightarrow 1 \mathrm{Zn} ; 24 g 1: 0.67 \mathrm{Pt}, 0.33 \mathrm{Zn} \rightarrow 1 \mathrm{Pt}\end{array}$ \\
\hline $\mathrm{Zn}_{80.0} \mathrm{Pt}_{20.0}$ & 12,10 & 84,20 & 16e3: $0.88 \mathrm{Zn}, 0.12 \mathrm{Pt} \rightarrow 1 \mathrm{Zn} ; 16 e 5: 0.62 \rightarrow 1 \mathrm{Zn} ; 48 h 1: 0.87 / 0.12 \rightarrow 1 \mathrm{Zn}$ \\
\hline $\mathrm{Zn}_{78.5} \mathrm{Pd}_{14.0} \mathrm{Al}_{7.5}$ & $12,16,3$ & $82,14,4$ & 16e5: $0.81 \mathrm{Zn}, 0.19 \mathrm{Al} \rightarrow 1 \mathrm{Zn} ; 48 h 4: 0.77 \mathrm{Zn}, 0.23 \mathrm{Al} \rightarrow 1 \mathrm{Zn}$ \\
\hline $\mathrm{Cu}_{73.9} \mathrm{Sn}_{26.1}$ & 11,4 & 82,22 & 16e7: $0.75 \rightarrow 1 \mathrm{Cu} ; 48 h 1: 0.75 \rightarrow 1 \mathrm{Cu}(\mathrm{Ni}$ sites unspecified) \\
\hline $\mathrm{Cu}_{78.6} \mathrm{Sn}_{21.4}$ & 11,4 & 82,22 & $16 e 7: 0.75 \rightarrow 1 \mathrm{Cu}$ \\
\hline $\mathrm{Cu}_{79.8} \mathrm{Sn}_{20.2}$ & 11,4 & 82,22 & $\begin{array}{l}\text { 16e8: } 0.83 \mathrm{Cu}, 0.17 \mathrm{Sn} \rightarrow 1 \mathrm{Cu} ; 24 f 1: 0.81 \mathrm{Cu}, 0.19 \mathrm{Sn} \rightarrow 1 \mathrm{Cu} \\
\text { 48h4: } 0.86 \mathrm{Sn}, 0.14 \mathrm{Cu} \rightarrow 1 \mathrm{Sn}\end{array}$ \\
\hline \multicolumn{4}{|c|}{ Subtype I/II (3 structures) } \\
\hline $\mathrm{Zn}_{89.1} \operatorname{Ir}_{10.9}$ & 12,9 & 93,8 & $\begin{array}{l}4 b: 0.51 \rightarrow 1 \mathrm{Zn} ; 16 e 1: 0.82 \mathrm{Zn}, 0.18 \mathrm{Ir} \rightarrow 1 \mathrm{Zn} ; 16 e 7: 0.64 \rightarrow 1 \mathrm{Zn} ; 24 f 2: 0.62 \rightarrow 1 \mathrm{Zn} \\
24 g 1: 0.62 \mathrm{Zn}, 0.38 \mathrm{Ir} \rightarrow 1 \mathrm{Zn} ; 48 h 6: 0.33 \mathrm{Zn} \rightarrow 0\end{array}$ \\
\hline $\mathrm{Zn}_{90.5} \operatorname{Ir}_{9.5}$ & 12,9 & 95,8 & $\begin{array}{l}4 b: 0.63 \rightarrow 1 \mathrm{Zn} ; 4 c: 0.25 \mathrm{Ir} \rightarrow 0 ; 16 e 1: 0.91 \mathrm{Zn}, 0.09 \mathrm{Ir} \rightarrow 1 \mathrm{Zn} ; 16 e 6: 0.62 / 0.25 \rightarrow 1 \mathrm{Zn} \\
\text { 16e7: } 0.45 \mathrm{Zn} \rightarrow 0 ; 24 f 2: 0.36 \mathrm{Zn} \rightarrow 0 ; 24 g 1: 0.84 \mathrm{Zn}, 0.16 \mathrm{Ir} \rightarrow 1 \mathrm{Zn} ; \\
\text { 48h3: } 0.62 / 0.40 \rightarrow 1 \mathrm{Zn} ; 48 h 6: 0.57 \rightarrow 1 \mathrm{Zn}\end{array}$ \\
\hline $\mathrm{Zn}_{91.1} \operatorname{Ir}_{8.9}$ & 12,9 & 95,8 & $\begin{array}{l}4 b: 0.70 \rightarrow 1 \mathrm{Zn} ; 4 c: 0.41 \mathrm{Ir} \rightarrow 0 ; 16 e 1: 0.98 \mathrm{Zn}, 0.02 \mathrm{Ir} \rightarrow 1 \mathrm{Zn} ; 16 e 6: 0.60 / 0.30 \rightarrow 1 \mathrm{Zn} ; \\
16 e 7: 0.42 \mathrm{Zn} \rightarrow 0 ; 24 f 2: 0.29 \mathrm{Zn} \rightarrow 0 ; 24 g 1: 0.90 \mathrm{Zn}, 0.10 \mathrm{Ir} \rightarrow 1 \mathrm{Zn} ; 48 h 6: 0.62 \rightarrow 1 \mathrm{Zn}\end{array}$ \\
\hline \multicolumn{4}{|c|}{ Subtype II (14 structures) } \\
\hline $\mathrm{Na}_{86.3} \mathrm{Tl}_{13.7}$ & 9,13 & 88,14 & $24 f 1: 0.94 \rightarrow 1 \mathrm{Na}$ \\
\hline $\mathrm{Sc}_{86.3} \mathrm{Os}_{13.7}$ & 11,14 & 88,14 & - \\
\hline $\mathrm{Mg}_{87.3} \mathrm{Ru}_{12.7}$ & 8,14 & 88,14 & 16e4: $0.75 \mathrm{Ru}, 0.25 \mathrm{Mg} \rightarrow 1 \mathrm{Ru} ; 16 e 6: 0.67 \mathrm{Mg}, 0.33 \mathrm{Ru} \rightarrow 1 \mathrm{Mg}$ \\
\hline $\mathrm{Mg}_{86.3} \mathrm{Rh}_{13.7}$ & 8,15 & 88,14 & - \\
\hline $\mathrm{Mg}_{87.9} \operatorname{Ir}_{12.1}$ & 8,9 & 87,12 & - \\
\hline $\mathrm{Mg}_{85.9} \mathrm{Pd}_{14.1}$ & 8,16 & 87,12 & 4b: $0.97 \rightarrow 1 \mathrm{Mg} ; 16 e 2: 0.51 \mathrm{Mg}, 0.49 \mathrm{Pd} \rightarrow 1 \mathrm{Mg}$ \\
\hline $\mathrm{Mg}_{86.3} \operatorname{Ir}_{13.7}$ & 8,9 & 88,14 & - \\
\hline $\mathrm{Zn}_{95.3} \mathrm{Mo}_{4.7}$ & 12,12 & 100,5 & $\begin{array}{l}4 a: 0.65 \rightarrow 1 \mathrm{Mo} ; 4 c: 0.57 \mathrm{Mo}, 0.43 \mathrm{Zn} \rightarrow 1 \mathrm{Mo} ; 16 e 5: 0.35 \rightarrow 1 \mathrm{Zn} ; 16 e 6: 0.44 \rightarrow 1 \mathrm{Zn} \\
24 g 1: 0.70 \rightarrow 1 \mathrm{Zn}\end{array}$ \\
\hline $\mathrm{Al}_{65.3} \mathrm{Cu}_{18.1} \mathrm{Cr}_{16.6}$ & $3,12,17$ & $72,18,12$ & $\begin{array}{l}\text { 4b: } 0.28 \mathrm{Cu} \rightarrow 0 ; 16 e 4: 0.74 \mathrm{Cr}, 0.26 \mathrm{Cu} \rightarrow 1 \mathrm{Cr} ; 16 e 5: 0.79 \mathrm{Al}, 0.21 \mathrm{Cu} \rightarrow 1 \mathrm{Al} \\
24 g 2: 0.58 \mathrm{Al}, 0.42 \mathrm{Cu} \rightarrow 1 \mathrm{Al} ; 48 h 4: 0.85 \mathrm{Al}, 0.15 \mathrm{Cu} \rightarrow 1 \mathrm{Al} ; 48 h 6: 0.5 \rightarrow 1 \mathrm{Cu}\end{array}$ \\
\hline $\mathrm{Zn}_{86.6} \mathrm{Fe}_{13.4}$ & 12,14 & 89,15 & $\begin{array}{l}\text { 4c: } 0.68 \mathrm{Fe} / \mathrm{Ni} \rightarrow 1 \mathrm{Fe} ; 16 e 3: 1 \mathrm{Fe} / \mathrm{Ni} \rightarrow 1 \mathrm{Fe} ; 16 e 6: 0.53 \mathrm{Fe} / \mathrm{Ni}, 0.25 \mathrm{Fe} / \mathrm{Ni} \rightarrow 1 \mathrm{Fe} \\
24 g 1: 1 \mathrm{Fe} / \mathrm{Ni} \rightarrow 1 \mathrm{Fe}\end{array}$ \\
\hline $\mathrm{Zn}_{86.6} \mathrm{Ni}_{13.4}$ & 12,16 & 89,15 & $\begin{array}{l}\text { 4c: } 0.68 \mathrm{Fe} / \mathrm{Ni} \rightarrow 1 \mathrm{Ni} ; 16 e 3: 1 \mathrm{Fe} / \mathrm{Ni} \rightarrow 1 \mathrm{Ni} ; 16 e 6: 0.53 \mathrm{Fe} / \mathrm{Ni}, 0.25 \mathrm{Fe} / \mathrm{Ni} \rightarrow 1 \mathrm{Ni} \\
24 g 1: 1 \mathrm{Fe} / \mathrm{Ni} \rightarrow 1 \mathrm{Ni}\end{array}$ \\
\hline $\mathrm{Zn}_{78.4} \mathrm{Fe}_{21.6}$ & 14,12 & 76,26 & 16e1: $1 \mathrm{Fe} / \mathrm{Zn} \rightarrow 1 \mathrm{Fe} ; 16 e 4: 1 \mathrm{Fe} / \mathrm{Zn} \rightarrow 1 \mathrm{Fe} ; 16 e 5: 1 \mathrm{Fe} / \mathrm{Zn} \rightarrow 1 \mathrm{Fe}$ \\
\hline $\mathrm{In}_{54.5} \mathrm{Pd}_{29.3} \mathrm{Ce}_{16.3}$ & $13,16,12$ & $67,36,20$ & 48h3: 0.66/0.34 $\rightarrow 1 \mathrm{Pd} ; 48 h 6: 0.68 / 0.32 \rightarrow 1 \mathrm{In}$ \\
\hline $\mathrm{Zn}_{67.5} \mathrm{Ce}_{16.7} \mathrm{Mg}_{15.8}$ & $12,12,8$ & $81,20,19$ & - \\
\hline \multicolumn{4}{|c|}{ Subtype III (5 structures) } \\
\hline $\mathrm{Mg}_{83.6} \mathrm{Gd}_{16.4}$ & 8,9 & 90,22 & 16e6: $0.70 \rightarrow 1 \mathrm{Mg} ; 24 g 2: 0.86 \rightarrow 1 \mathrm{Mg} ; 48 h 2: 0.68 \mathrm{Gd}, 0.32 \mathrm{Mg} \rightarrow 1 \mathrm{Gd}$ \\
\hline $\mathrm{Cd}_{80.4} \mathrm{Sm}_{19.6}$ & 12,11 & 90,22 & - \\
\hline $\mathrm{Al}_{63.6} \mathrm{Ta}_{36.4}$ & 3,11 & 72,39 & $\begin{array}{l}\text { 16e6: } 0.33 / 0.33 / 0.33 \rightarrow 1 \mathrm{Al} ; 24 g 1: 0.5 / 0.5 \rightarrow 1 \mathrm{Al} ; 48 h 2: 0.79 / 0.21 \rightarrow 1 \mathrm{Al} ; \\
\text { 48h3: } 0.82 / 0.18 \rightarrow 1 \mathrm{Ta} ; 48 h 4: 0.71 / 0.14 / 0.14 \rightarrow 1 \mathrm{Al} ; 48 h 6: 0.88 \mathrm{Al}, 0.12 \mathrm{Ta} \rightarrow 1 \mathrm{Al}\end{array}$ \\
\hline $\mathrm{Mg}_{82.4} \mathrm{Y}_{9.0} \mathrm{Ce}_{8.6}$ & $8,11,12$ & $90,4,18$ & $\begin{array}{l}\text { 4b: } 0.62 \mathrm{Ce}, 0.38 \mathrm{Y} \rightarrow 1 \mathrm{Ce} ; 4 c: 0.62 \mathrm{Ce}, 0.38 \mathrm{Y} \rightarrow 1 \mathrm{Ce} ; 16 e 4: 0.62 \mathrm{Ce}, 0.38 \mathrm{Y} \rightarrow 1 \mathrm{Ce} \\
16 e 6: 0.7 \rightarrow 1 \mathrm{Mg} ; 24 g 1: 0.86 \rightarrow 1 \mathrm{Mg} ; 48 h 2: 0.48 \mathrm{Ce}, 0.30 \mathrm{Y}, 0.23 \mathrm{Mg} \rightarrow 1 \mathrm{Ce}\end{array}$ \\
\hline $\mathrm{Na}_{49.2} \mathrm{Ba}_{28.8} \mathrm{Li}_{22.0}$ & $7,10,3$ & $58,38,26$ & - \\
\hline \multicolumn{4}{|c|}{ Subtype IV (1 structure) } \\
\hline $\mathrm{Na}_{49.1} \mathrm{Sn}_{26.3} \operatorname{In}_{24.6}$ & $1,4,3$ & $56,30,28$ & 48h6: $0.83 \mathrm{In}, 0.17 \mathrm{Sn} \rightarrow 1 \mathrm{In}$ \\
\hline
\end{tabular}




\section{Table 28}

Details on the performed $a b$ initio calculations for structures of space group $F d \overline{3} m$. The data are given as described for Table 27 .

\begin{tabular}{l|lll}
\hline Structure & $e$ atom & atoms/u.c. & Applied idealization steps \\
\hline $\mathrm{In}_{70.7} \mathrm{~K}_{29.3}$ & 13,9 & 82,34 & - \\
$\mathrm{Ga}_{50.0} \mathrm{Na}_{29.3} \mathrm{In}_{20.7}$ & $13,7,13$ & $58,34,24$ & - \\
$\mathrm{Ga}_{48.9} \mathrm{Na}_{30.4} \mathrm{Cd}_{20.7}$ & $13,7,12$ & $48,36,32$ & $8 b: 0.49 \rightarrow 1 \mathrm{Na} ; 32 e 1: 0.85 \mathrm{Cd}, 0.15 \mathrm{Ga} \rightarrow 1 \mathrm{Cd} ; 96 g 2: 0.71 \mathrm{Cd}, 0.29 \mathrm{Ga} \rightarrow 1 \mathrm{Cd}$ \\
$\mathrm{Ga}_{63.0} \mathrm{Na}_{31.0} \mathrm{Ag}_{6.0}$ & $13,7,11$ & $48,36,32$ & $32 e 1: 0.67 \mathrm{Ga}, 0.33 \mathrm{Ag} \rightarrow 1 \mathrm{Ag} ; 96 g 2: 0.82 \mathrm{Ga}, 0.18 \mathrm{Ag} \rightarrow 1 \mathrm{Ag}$ \\
$\mathrm{Ga}_{53.4} \mathrm{Li}_{31.0} \mathrm{Cu}_{8.6} \mathrm{In}_{6.9}$ & $13,3,17,-$ & $72,36,8,0$ & $9694: 0.58 \mathrm{Ga}, 0.42 \mathrm{Cu} \rightarrow 1 \mathrm{Ga}$ \\
$\mathrm{Ga}_{47.5} \mathrm{Mg}_{31.2} \mathrm{Cu}_{21.3}$ & $13,8,17$ & $56,36,24$ & $8 a: 0.56 \rightarrow 1 \mathrm{Mg} ; 32 e 1: 0.70 \rightarrow 1 \mathrm{Ga}$ \\
\hline $\mathrm{Zn}_{61.0} \mathrm{Ca}_{35.6} \mathrm{Ni}_{3.4}$ & $12,8,16$ & $72,42,4$ & - \\
\hline
\end{tabular}




\section{Electron localization function - (110) layers}
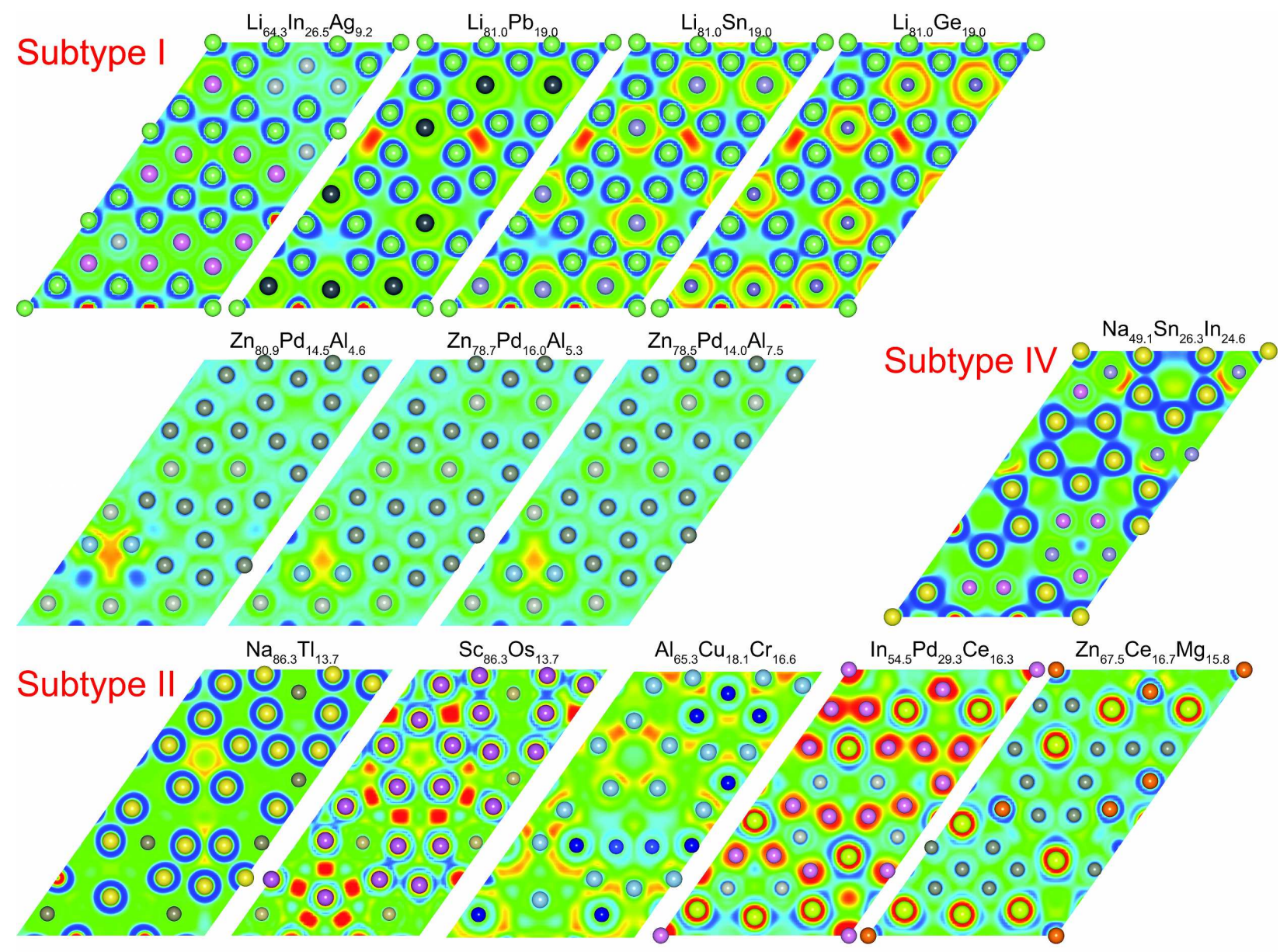

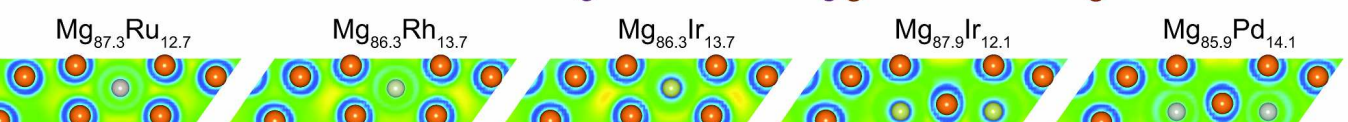

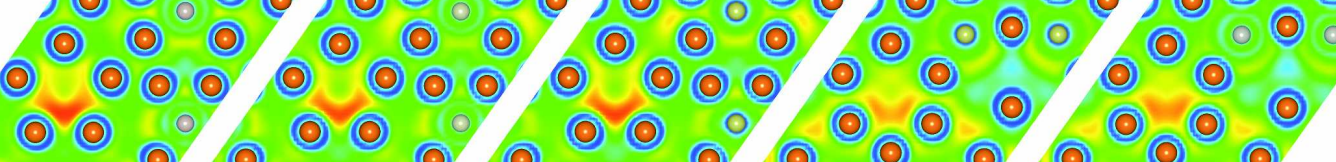

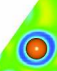

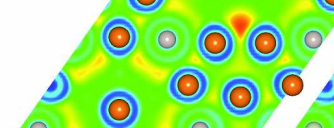

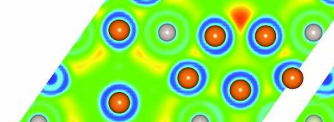
() (c) (c) ( () () (c) (C) () () (ㅇ) (๑) () () () () () ( $)$ (2) 0 () (ㅇ) (C) (C) (c) (-) (ㅇ)

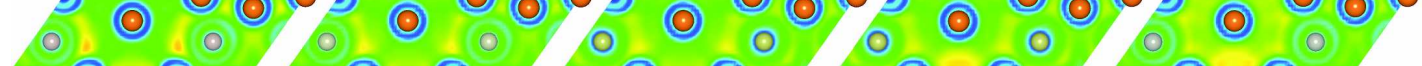

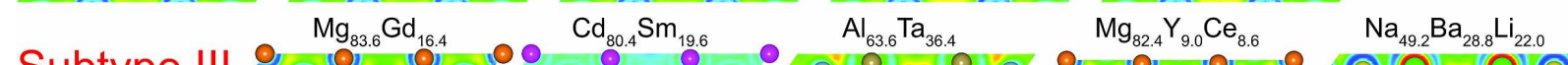

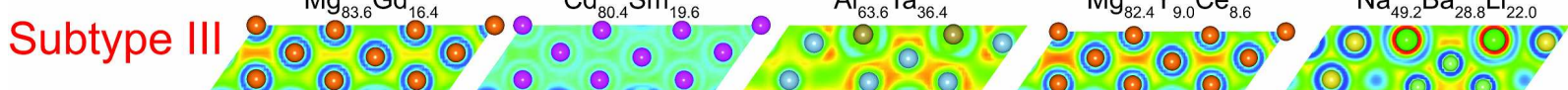

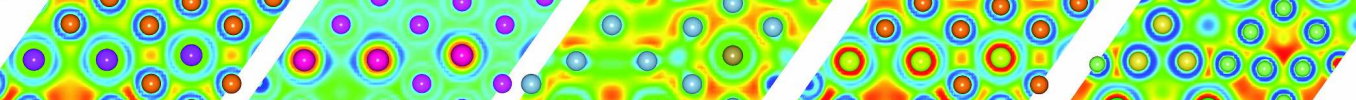

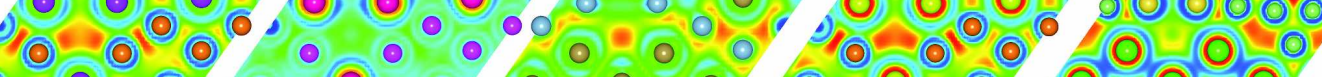

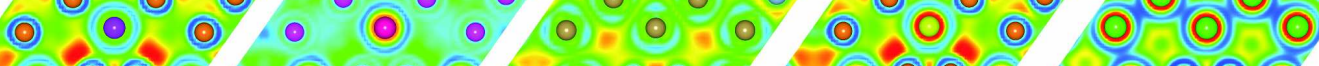

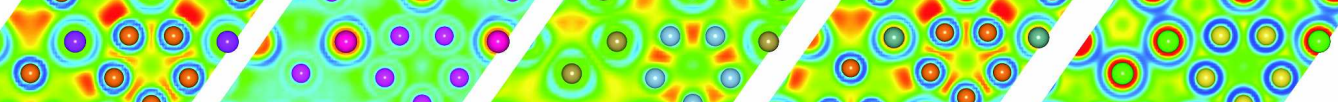

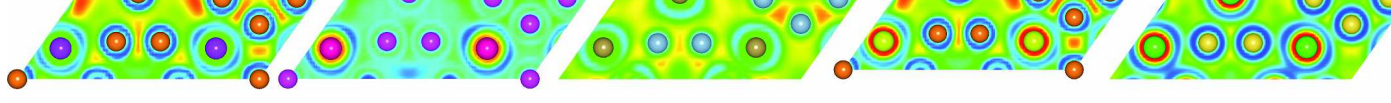

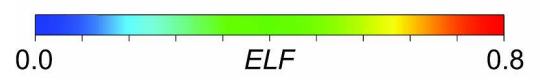

Figure 3

(110) layers of the ELFs of cubic complex intermetallics in the $F \overline{4} 3 m$ space group - structures with values $E L F>0.5$. 

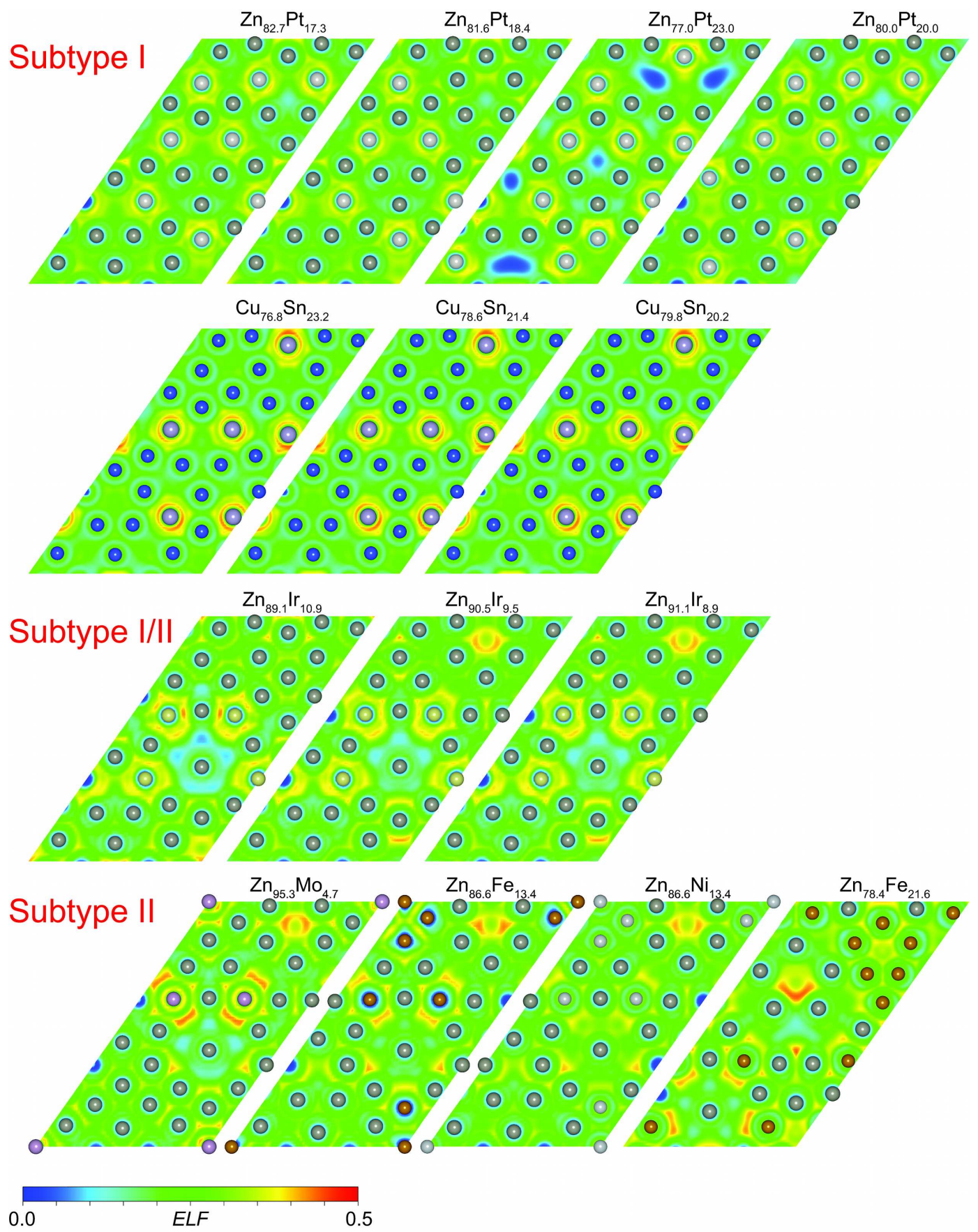

\section{Figure 4}

(110) layers of the ELFs of cubic complex intermetallics in the $F \overline{4} 3 m$ space group - structures only with values $E L F<0.5$. Due to structural ambiguities as reported, the $\mathrm{Cu}_{73.9} \mathrm{Sn}_{23.2} \mathrm{Ni}_{2.9}$ was simulated as $\mathrm{Cu}_{76.8} \mathrm{Sn}_{23.2}$ and is also inscribed in this way. Similarly, the structure of $\mathrm{Zn}_{86.6} \mathrm{Fe}_{6.7} \mathrm{Ni}_{6.7}$ was simulated in the variants $\mathrm{Zn}_{86.6} \mathrm{Fe}_{13.4}$ and $\mathrm{Zn}_{86.6} \mathrm{Ni}_{13.4}$. 


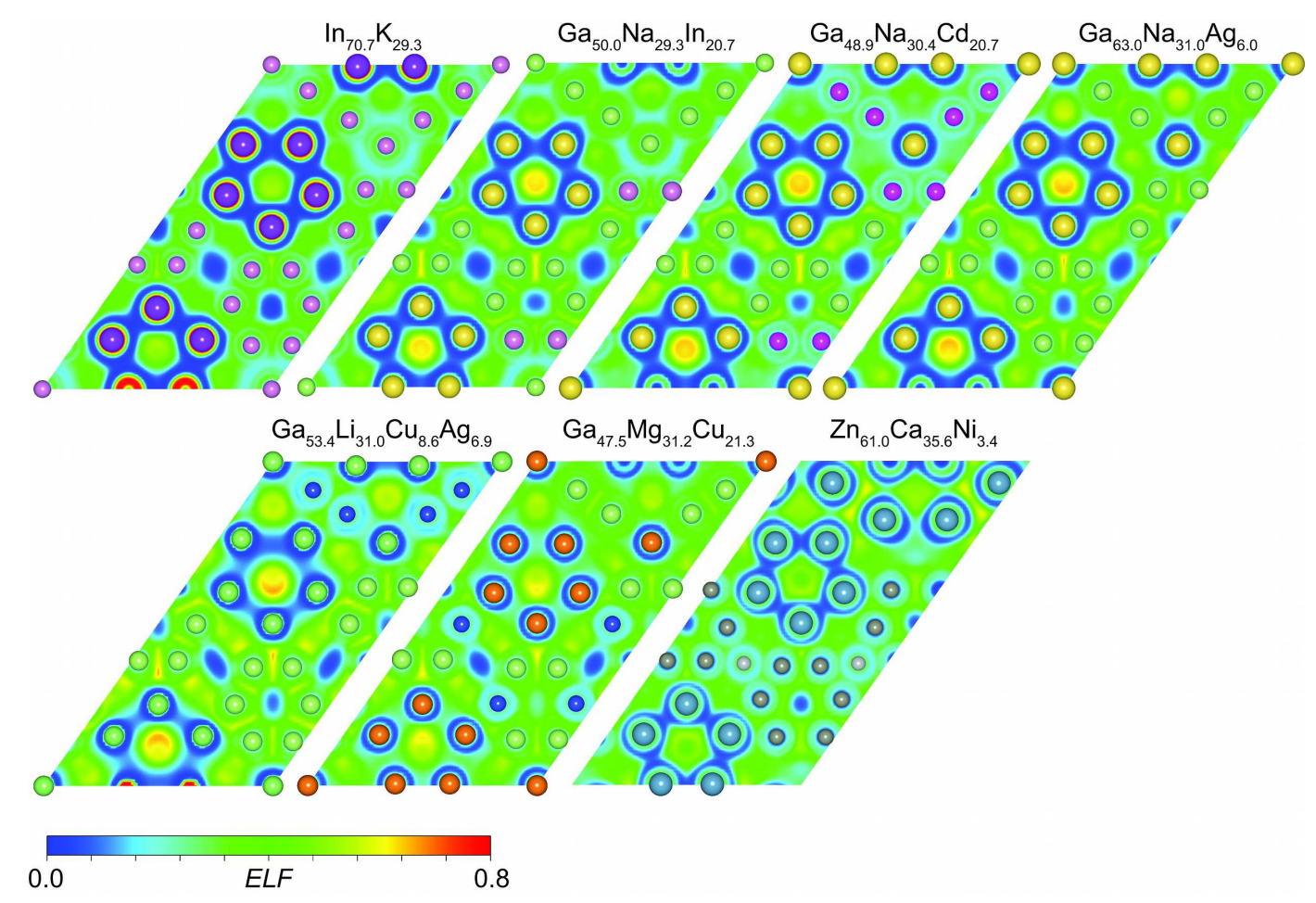

Figure 5

(110) layers of the ELFs of cubic complex intermetallics in the $F d \overline{3} m$ space group. The structures are labeled with their respective chemical formulas. 


\section{Bader charge analysis}

\section{Table 29}

Bader charges of cubic complex intermetallics $c F 464$ in space group $F \overline{4} 3 m,(3 \times 3 \times 3)$-fold superstructures of a common basic structure. Given are for all calculated structures the Bader charges, expressed by the electron difference with respect to neutral atoms, for the different atomic sorts. The structures $\mathrm{Cu}_{73.9} \mathrm{Sn}_{23.2} \mathrm{Ni}_{2.9}$ and $\mathrm{Zn}_{86.6} \mathrm{Fe}_{6.7} \mathrm{Ni}_{6.7}$ had to be calculated in the variants $\mathrm{Cu}_{76.8} \mathrm{Sn}_{23.2}$ and $\mathrm{Zn}_{86.6} \mathrm{Fe}_{13.4}$, as well as $\mathrm{Zn}_{86.6} \mathrm{Ni}_{13.4}$, respectively.

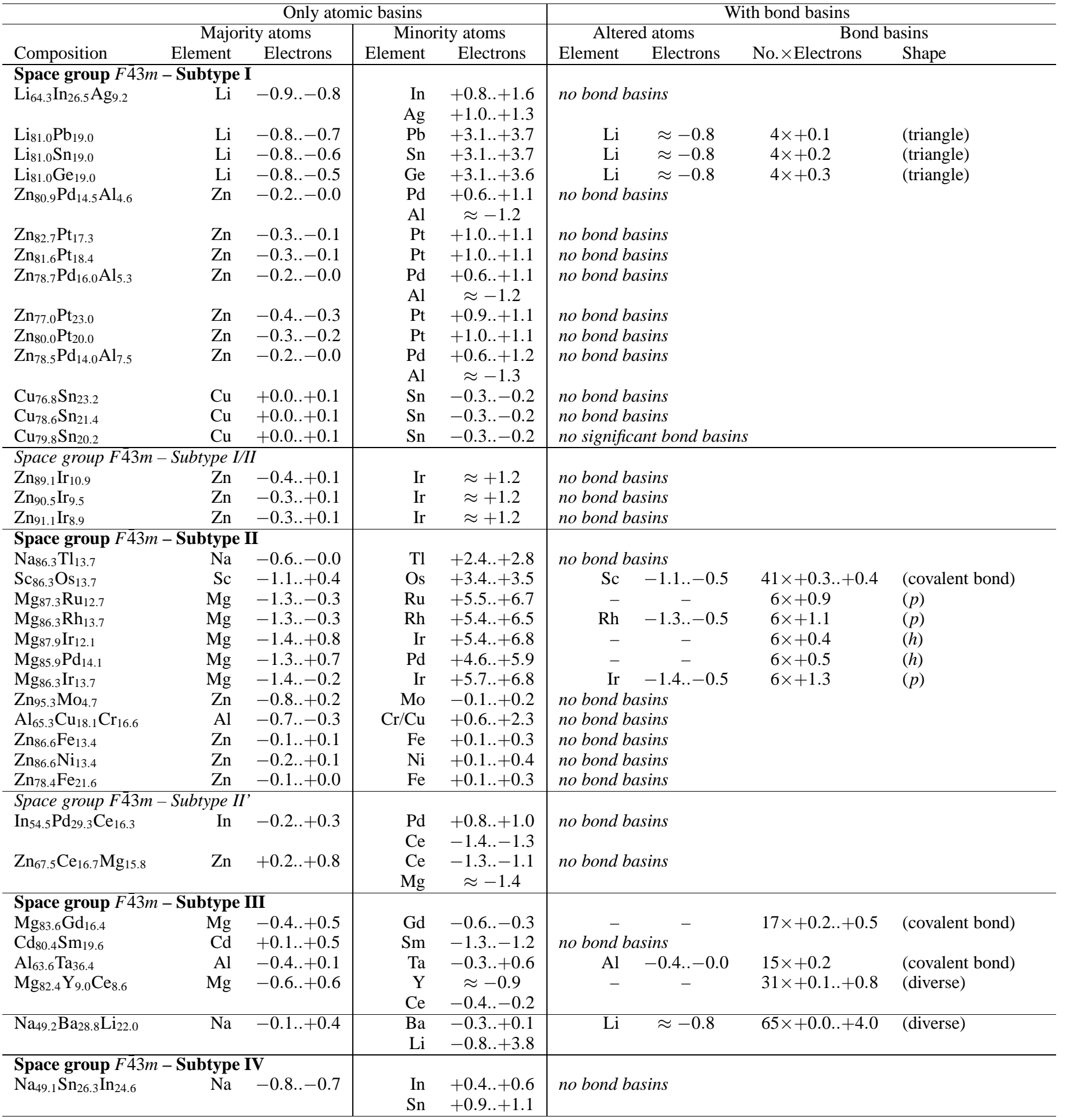


Table 30

Bader charges of cubic complex intermetallics $c F 464$ in space group $F d \overline{3} m,(3 \times 3 \times 3)$-fold superstructures of a common basic structure. Given are for all calculated structures the Bader charges, expressed by the electron difference with respect to neutral atoms, for the different atomic sorts.

\begin{tabular}{|c|c|c|c|c|c|c|c|}
\hline \multirow[b]{3}{*}{ Composition } & \multicolumn{4}{|c|}{ Only atomic basins } & \multicolumn{3}{|c|}{ With bond basins } \\
\hline & \multicolumn{2}{|c|}{ Majority atoms } & \multicolumn{2}{|c|}{ Minority atoms } & Altered atoms & \multicolumn{2}{|c|}{ Bond basins } \\
\hline & Element & Electrons & Element & Electrons & Element Electrons & No. $\times$ Electrons & Shape \\
\hline \multicolumn{8}{|l|}{ Space group $F d 3 m$} \\
\hline $\operatorname{In}_{70.7} \mathrm{~K}_{29.3}$ & In & $+0.0 . .+0.3$ & $\mathrm{~K}$ & $\approx-0.7$ & no bond basins & & \\
\hline \multirow[t]{2}{*}{$\mathrm{Ga}_{50.0} \mathrm{Na}_{29.3} \mathrm{In}_{20.7}$} & $\mathrm{Ga}$ & $+0.0 . .+0.3$ & $\mathrm{Na}$ & $\approx-0.8$ & no bond basins & & \\
\hline & & & In & $\approx+0.4$ & & & \\
\hline \multirow[t]{2}{*}{$\mathrm{Ga}_{48.9} \mathrm{Na}_{30.4} \mathrm{Cd}_{20.7}$} & $\mathrm{Ga}$ & $\approx+0.3$ & $\mathrm{Na}$ & $\approx-0.8$ & no bond basins & & \\
\hline & & & $\mathrm{Cd}$ & $\approx+0.4$ & & & \\
\hline \multirow[t]{2}{*}{$\mathrm{Ga}_{63.0} \mathrm{Na}_{31.0} \mathrm{Ag}_{6.0}$} & $\mathrm{Ga}$ & $+0.3 . .+0.4$ & $\mathrm{Na}$ & $\approx-0.8$ & no bond basins & & \\
\hline & & & $\mathrm{Ag}$ & $+0.4 . .+0.5$ & & & \\
\hline \multirow{2}{*}{$\mathrm{Ga}_{53.4} \mathrm{Li}_{31.0} \mathrm{Cu}_{8.6} \mathrm{In}_{6.9}$} & $\mathrm{Ga}$ & $+0.3 . .+0.4$ & $\mathrm{Li}$ & $\approx-0.9$ & no bond basins & & \\
\hline & & 05 & $\mathrm{Cu}$ & $\approx+0.6$ & houd havin & & \\
\hline $\mathrm{Ga}_{47.5} \mathrm{Mg}_{31.2} \mathrm{Cu}_{21.3}$ & Ua & $+0.5 . .+0.0$ & $\begin{array}{l}\mathrm{Mg} \\
\mathrm{Cu}\end{array}$ & $\begin{array}{l}\approx-1.5 \\
\approx+0.1\end{array}$ & no bona basins & & \\
\hline \multirow[t]{2}{*}{$\mathrm{Zn}_{61.0} \mathrm{Ca}_{35.6} \mathrm{Ni}_{3.4}$} & $\mathrm{Zn}$ & $+0.5 . .+1.0$ & $\mathrm{Ca}$ & $\approx-1.2$ & no bond basins & & \\
\hline & & & $\mathrm{Ni}$ & $\approx+0.4$ & & & \\
\hline
\end{tabular}




\section{Density of states}
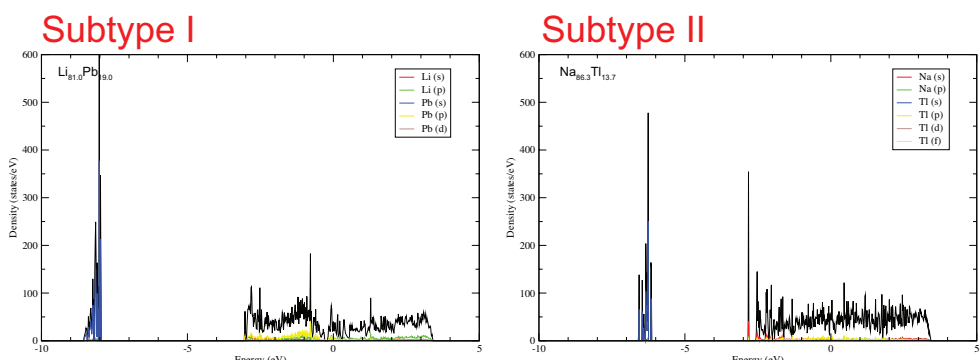

Subtype IV
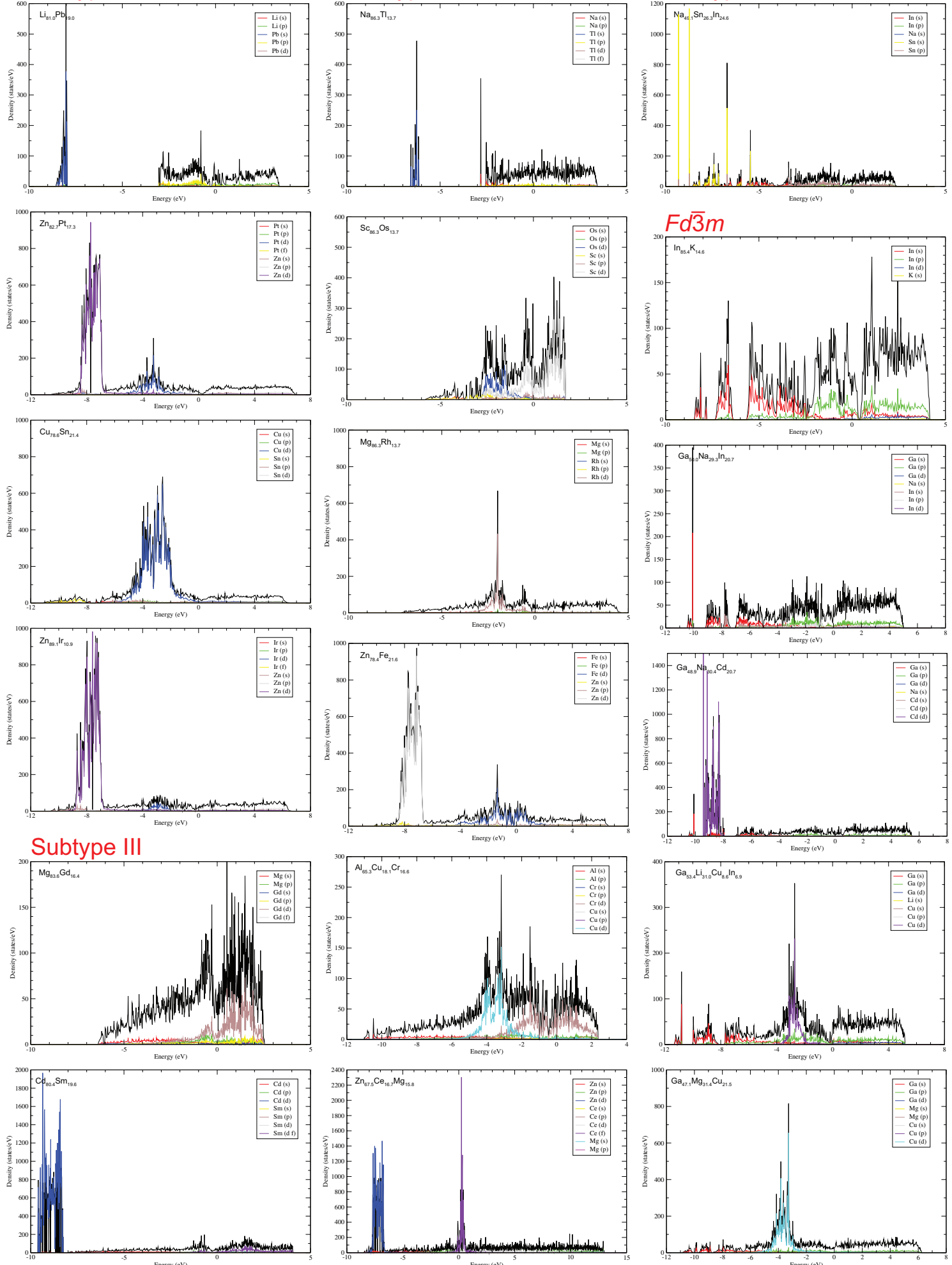

Figure 6

Density of states (DOS) graphs for representatives of the investigated structures $(c F(464-x))$. 